Homology, Homotopy and Applications, vol.11(1), 2009, pp.219-260

\title{
LOCAL AND STABLE HOMOLOGICAL ALGEBRA IN GROTHENDIECK ABELIAN CATEGORIES
}

\author{
DENIS-CHARLES CISINSKI AND FRÉDÉRIC DÉGLISE
}

\author{
(communicated by Brooke Shipley)
}

\begin{abstract}
We define model category structures on the category of chain complexes over a Grothendieck abelian category depending on the choice of a generating family, and we study their behaviour with respect to tensor products and stabilization. This gives convenient tools to construct and understand triangulated categories of motives.
\end{abstract}

\section{Contents}

2 Model category structures on complexes

3 Derived tensor product

4 Localization of derived categories

5 Presentations of the derived category

6 How to get compact generators

7 Stabilization and symmetric spectra

\section{Introduction}

This paper is an attempt to apply the general techniques of abstract homotopical algebra to describe derived categories of Grothendieck categories. Our motivation is to describe model categories of complexes of sheaves "as locally as possible", so that we can easily get total left derived functors (like the tensor product). The formalism we propose is made to stay close to something that looks like descent theory. Even though the results proved here are just applications of a general theory (Bousfield

This work is partially supported by ANR grant no. ANR-07-BLAN-0142

Received January 14, 2008, revised November 21, 2008; published on June 12, 2009.

2000 Mathematics Subject Classification: 18G55, 18G35, 18E15, 14F42.

Key words and phrases: stable model categories, Grothendieck abelian categories, derived functors, motivic complexes.

This article is available at http://intlpress.com/HHA/v11/n1/a11

Copyright (C) 2009, International Press. Permission to copy for private use granted. 
localization, symmetric spectra), our contribution describes these model structures with an emphasis on the specific properties in the setting of Grothendieck categories. As an example of properties that are not known for general model categories, we give explicit descriptions of fibrations after Bousfield localizations and prove that passing to symmetric spectra can preserve the monoid axiom of Schwede and Shipley. We also prove that any derived category of a Grothendieck category can be obtained as a localization of the derived category of modules over an additive category. This is used to give a sufficient condition for the derived category of a Grothendieck category to be compactly generated.

As an illustration, we give the construction of the categories of motivic complexes and motivic spectra over a regular base scheme together with their natural structures: derived inverse and direct image functors, derived tensor product, and twisted exceptional direct image functors for smooth morphisms. In this construction, smooth always means abusively smooth of finite type. This construction will be amplified and generalized in the forthcoming paper [6]. The results of this paper are also widely used to understand the notion of mixed Weil cohomology, which is introduced and studied in [7].

We wish to thank Marc Levine and the anonymous referee for useful comments and suggestions.

\section{Model category structures on complexes}

2.1.

Let $\mathcal{A}$ be an abelian category. If $E$ is an object of $\mathcal{A}$ and $n$ an integer, then we define $S^{n} E$ (resp. $\left.D^{n} E\right)$ as the acyclic complex concentrated in degree $n$ with $\left(S^{n} E\right)^{n}=E$ (resp. in degrees $n$ and $n+1$ with $\left(D^{n} E\right)^{n}=\left(D^{n} E\right)^{n+1}=E$ and as only a nontrivial differential the identity of $E$ ). We then have canonical inclusions $S^{n+1} E \longrightarrow D^{n} E$ induced by the identity of $E$. The complex $D^{n} E$ represents the functor

$$
\operatorname{Comp}(\mathcal{A})^{o p} \longrightarrow A b, \quad C \longmapsto \operatorname{Hom}_{\mathcal{A}}\left(E, C^{n}\right),
$$

and the complex $S^{n} E$ represents the functor

$$
\operatorname{Comp}(\mathcal{A})^{o p} \longrightarrow A b, \quad C \longmapsto \operatorname{Hom}_{\mathcal{A}}\left(E, Z^{n} C\right),
$$

where $Z^{n} C=\operatorname{ker}\left(d: C^{n} \longrightarrow C^{n+1}\right)$ denotes the object of $n$-cocycles of the complex $C$. We shall refer ourselves to [12] for general results and definitions on model categories, and to [11] for the definition of cellular model categories ${ }^{1}$ and for results on Bousfield localizations.

Theorem 2.1 (Beke). Let $\mathcal{A}$ be a Grothendieck abelian category. The category of complexes $\operatorname{Comp}(\mathcal{A})$ is a proper cellular model category with quasi-isomorphisms as weak equivalences and the monomorphisms as cofibrations.

\footnotetext{
${ }^{1}$ This notion is useful to ensure the existence of left Bousfield localizations. For such a purpose, we can work instead with Jeff Smith's notion of combinatorial model category [3]. The notion of combinatorial model category might be seen as slightly simpler than the notion of cellular model category, but it is very easy to check that the model structures considered in these notes are both cellular and combinatorial, so we invite the reader to consider his/her favourite notion.
} 
Proof. See [3, Proposition 3.13] for the construction of this model category structure. The fact that this model category is cellular comes from the fact that any monomorphism in a Grothendieck category is effective, and that any object in a Grothendieck category is compact (in fact accessible according to [1]). Remark that fibrations are, in particular, epimorphisms. This is proved as follows: For any object $E$ in $\mathcal{A}$ and any integer $n$, the complex $D^{n} E$ is acyclic. Hence any fibration has the right lifting property with respect to the inclusions $0 \longrightarrow D^{n} E$. This lifting property for a map $p: C \longrightarrow D$ is equivalent to saying that for any object $E$ of $\mathcal{A}$ and any integer $n$, the map $\operatorname{Hom}_{\mathcal{A}}\left(E, C^{n}\right) \longrightarrow \operatorname{Hom}_{\mathcal{A}}\left(E, D^{n}\right)$ is surjective. This implies, in particular, that $p$ is degreewise split, hence that $p$ is an epimorphism. Properness now comes from classical homological algebra as follows. Let

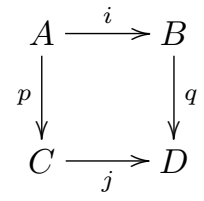

be a pushout (resp. a pullback) square of complexes in $\mathcal{A}$. Suppose that $i$ (resp. $q$ ) is a cofibration (resp. fibration). As $i$ (resp. $q$ ) is a monomorphism (resp. an epimorphism), the same is true for $j$ (resp. for $p$ ). Moreover, it is obvious that the cokernels of $i$ and $j$ (resp. the kernels of $p$ and $q$ ) are canonically isomorphic, which implies that we have a long exact sequence of shape:

$$
H^{n}(A) \longrightarrow H^{n}(B) \oplus H^{n}(C) \longrightarrow H^{n}(D) \longrightarrow H^{n+1}(A) .
$$

We deduce then immediately that if $p$ (resp. $j$ ) is a quasi-isomorphism, then so is $q$ (resp. $i$ ), which is what we wanted to prove.

\section{2 .}

The above model category structure will be called the injective model structure. The fibrations of this model structure will be called the injective fibrations, and the fibrant objects the injectively fibrant objects.

The injective model structure gives a powerful tool to construct right derived functors (well defined on unbounded complexes). To define left derived functors on unbounded complexes, we have to consider other model structures depending on the choice of a set of generators of $\mathcal{A}$. We shall restrict ourselves to a special situation that will occur in the cases we are interested in. This can be well formulated using the intuition of descent theory.

Definition 2.2. Let $\mathcal{A}$ be a Grothendieck category.

If $\mathcal{G}$ is an essentially small set of objects of $\mathcal{A}$, then we define a morphism in $\operatorname{Comp}(\mathcal{A})$ to be a $\mathcal{G}$-cofibration if it is contained in the smallest class of maps in $\operatorname{Comp}(\mathcal{A})$ closed under pushouts, transfinite compositions and retracts, generated by the inclusions $S^{n+1} E \longrightarrow D^{n} E$, for any integer $n$ and any $E$ in $\mathcal{G}$. A chain complex $C$ is $\mathcal{G}$-cofibrant if the map $0 \longrightarrow C$ is a $\mathcal{G}$-cofibration. For example, if $C$ is a bounded above complex such that for any integer $n, C^{n}$ is a direct sum of elements of $\mathcal{G}$, then $C$ is $\mathcal{G}$-cofibrant. 
A chain complex $C$ in $\mathcal{A}$ is $\mathcal{G}$-local, or simply local if no confusion can arise from the context, if for any $E$ in $\mathcal{G}$ and integer $n$, the canonical map

$$
\operatorname{Hom}_{K(\mathcal{A})}(E[n], C) \longrightarrow \operatorname{Hom}_{D(\mathcal{A})}(E[n], C)
$$

is an isomorphism.

If $\mathcal{H}$ is a small family of complexes in $\mathcal{A}$, then an object $C$ of $\operatorname{Comp}(\mathcal{A})$ is $\mathcal{H}$-flasque (or simply flasque) if for any integer $n$ and any $H$ in $\mathcal{H}$, the group $\operatorname{Hom}_{K(\mathcal{A})}(H, C[n])$ vanishes.

A descent structure on a Grothendieck category $\mathcal{A}$ is a couple $(\mathcal{G}, \mathcal{H})$, where $\mathcal{G}$ is an essentially small set of generators of $\mathcal{A}$, and $\mathcal{H}$ is an essentially small set of $\mathcal{G}$-cofibrant acyclic complexes such that any $\mathcal{H}$-flasque complex is $\mathcal{G}$-local.

Example 2.3. Let $\mathcal{S}$ be an essentially small Grothendieck site and $\mathcal{R}$ a sheaf of rings on $\mathcal{S}$. Then the category $\mathcal{R}$-Mod of sheaves of $\mathcal{R}$-modules is a Grothendieck category. For an object $X$ of $\mathcal{S}$, denote by $\mathcal{R}(X)$ the free sheaf of $\mathcal{R}$-modules generated by $X$. The family $\mathcal{G}_{\mathcal{S}}$ of the $\mathcal{R}(X)$ 's form a generating set for $\mathcal{R}$-Mod. If $\mathcal{X}$ is a hypercover of $X$, then the simplicial $\mathcal{R}$-module $\mathcal{R}(\mathcal{X})$ can be seen as a complex of $\mathcal{R}$-modules with the differentials given by the alternating sum of the face operators. For a hypercovering $\mathcal{X}$ of an object $X$, denote by $\widetilde{\mathcal{R}}(\mathcal{X})$ the cone of the structural map $\mathcal{R}(\mathcal{X}) \longrightarrow \mathcal{R}(X)$. The complexes $\mathcal{R}(X), \mathcal{R}(\mathcal{X})$ and $\widetilde{\mathcal{R}}(\mathcal{X})$ are then $\mathcal{G}_{\mathcal{S}}$-cofibrant. Let $\mathcal{H}_{\mathcal{S}}$ be the family of all the complexes of the form $\widetilde{\mathcal{R}}(\mathcal{X})$ for any hypercover $\mathcal{X}$ of an object $X$ of $\mathcal{S}$. Then the couple $\left(\mathcal{G}_{\mathcal{S}}, \mathcal{H}_{\mathcal{S}}\right)$ is a descent structure on the category $\mathcal{R}$-Mod. One way to prove this comes from Verdier's computation of hypercohomology; see [1, Exposé V] and [4]. If $X$ is an object of $\mathcal{S}$ and $C$ is a complex of $\mathcal{R}$-modules, then we have the formula

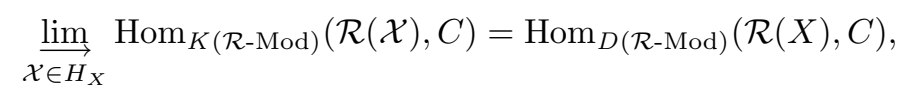

where $H_{X}$ denotes the filtering category of hypercoverings of $X$ up to simplicial homotopy (and $\operatorname{Hom}_{D(\mathcal{R} \text {-Mod) }}(\mathcal{R}(X), C)$ is nothing else but the hypercohomology of $X$ with coefficients in $C)$. We deduce that if $C$ is $\mathcal{H}_{\mathcal{S}}$-flasque, as we have distinguished triangles in $K(\mathcal{R}$-Mod) of shape

$$
\mathcal{R}(\mathcal{X}) \longrightarrow \mathcal{R}(X) \longrightarrow \widetilde{\mathcal{R}}(\mathcal{X}) \longrightarrow \mathcal{R}(\mathcal{X})[1]
$$

for any $\mathcal{X}$ in $H_{X}$, then we have the isomorphisms

$$
\operatorname{Hom}_{K(\mathcal{R} \text {-Mod })}(\mathcal{R}(X), C)=\operatorname{Hom}_{K(\mathcal{R} \text {-Mod })}(\mathcal{R}(\mathcal{X}), C) .
$$

As $H_{X}$ is filtering, we deduce that the canonical map

$$
\operatorname{Hom}_{K(\mathcal{R} \text {-Mod) }}(\mathcal{R}(X), C) \longrightarrow \varliminf_{\mathcal{X} \in H_{X}} \operatorname{Hom}_{K(\mathcal{R} \text {-Mod) }}(\mathcal{R}(\mathcal{X}), C)
$$

is an isomorphism. Hence $C$ is $\mathcal{G}_{\mathcal{S}}$-local.

This example generates new ones: let $\mathcal{A}$ be a Grothendieck abelian category endowed with a functor

$$
U: \mathcal{A} \longrightarrow \mathcal{R} \text {-Mod }
$$

which preserves colimits and limits, and which is conservative. Then the functor $U$ 
has a left adjoint

$$
F: \mathcal{R}-\operatorname{Mod} \longrightarrow \mathcal{A}
$$

and we can see that $\left(F\left(\mathcal{G}_{\mathcal{S}}\right), F\left(\mathcal{H}_{\mathcal{S}}\right)\right)$ is a descent structure on $\mathcal{A}$.

A natural example of this shape is the following: Let $\mathcal{S}=X_{\text {Zar }}$ be the small Zariski site of a quasi-separated and quasi-compact scheme, and let $\mathcal{R}=\mathcal{O}_{X}$ be the structural sheaf of rings on $X$. We can consider the Grothendieck abelian category $\mathcal{A}=\mathrm{QCoh}(X)$ of quasi-coherent $\mathcal{O}_{X}$-modules and see that the forgetful functor $U: \mathrm{QCoh}(X) \longrightarrow \mathcal{O}_{X}$-Mod preserves colimits and limits, and is conservative.

Another example which fits in this kind of presentation comes from the theory of algebraic cycles.

Example 2.4. Let $S$ be a noetherian regular scheme, and $\mathcal{N}_{S}^{t r}$ be the category of Nisnevich sheaves with transfers over $S$ defined in $[8,4.2 .3]$. Let $\mathcal{G}_{S}$ be the category of sheaves with transfers of type $L_{S}(X)$ for any smooth $S$-scheme $X$. Then it follows from Proposition 4.2 .8 of loc. cit. that $\mathcal{A}_{S}$ is a Grothendieck abelian category and that $\mathcal{G}_{S}$ is a set of generators. We denote by $\mathcal{H}_{S}$ the set of complexes obtained as the cones of the maps $L_{S}(\mathcal{X}) \longrightarrow L_{S}(X)$, where $X$ is any smooth $S$-scheme and $\mathcal{X}$ is any Nisnevich hypercovering of $X$. From the example above and from Propositions 4.2.5 and 4.2 .9 of loc. cit., it follows that $\left(\mathcal{G}_{S}, \mathcal{H}_{S}\right)$ is a descent structure on $\mathcal{N}_{S}^{t r}$.

Theorem 2.5. Let $\mathcal{A}$ be a Grothendieck category endowed with a descent structure $(\mathcal{G}, \mathcal{H})$. Then the category $\operatorname{Comp}(\mathcal{A})$ is a proper cellular model category with quasiisomorphisms as weak equivalences, and $\mathcal{G}$-cofibrations as cofibrations. Furthermore, for a complex $C$ in $\mathcal{A}$, the following conditions are equivalent:

(i) $C$ is fibrant;

(ii) $C$ is $\mathcal{H}$-flasque;

(iii) $C$ is $\mathcal{G}$-local.

This model structure on the category $\operatorname{Comp}(\mathcal{A})$ will be called the $\mathcal{G}$-model structure.

Remark 2.6. It is possible to give a nice description of the fibrations for this model category structure; see Corollary 5.5.

\section{3.}

The proof of this theorem will be completed by the results below. We first have to fix some notation.

We define $I$ to be the set of inclusions of the form $S^{n+1} E \longrightarrow D^{n} E$ for any integer $n$ and any $E$ in $\mathcal{G}$.

If $C$ is a complex, then $\operatorname{Cyl}(C)$ is the complex defined by

$$
\operatorname{Cyl}(C)^{n}=C^{n} \oplus C^{n+1} \oplus C^{n}
$$

with the differential

$$
d(x, y, z)=(d x-y,-d y, y+d z) .
$$

The projection $\sigma: \operatorname{Cyl}(C) \longrightarrow C$, defined by the formula $\sigma(x, y, z)=x+z$, is a quasiisomorphism. We also have a canonical inclusion $\left(i_{0}, i_{1}\right): C \oplus C \longrightarrow \mathrm{Cyl}(C)$ defined by $i_{0}(x)=(x, 0,0)$ and $i_{1}(z)=(0,0, z)$, so that the composite $\sigma\left(i_{0}, i_{1}\right)$ is equal to the 
codiagonal from $C \oplus C$ to $C$. It is an easy exercise to check that to define a chain homotopy between two maps of complexes $u_{0}$ and $u_{1}$ from $C$ to $C^{\prime}$ is equivalent to defining a map $h$ from $\operatorname{Cyl}(C)$ to $C^{\prime}$ such that $h i_{e}=u_{e}$ for $e=0,1$.

We define at last $J=J^{\prime} \cup J^{\prime \prime}$, where $J^{\prime}$ is the set of maps $0 \longrightarrow D^{n} E$ for any integer $n$, any $E$ in $\mathcal{G}$, and $J^{\prime \prime}$ is the set of maps $H \oplus H[n] \longrightarrow \operatorname{Cyl}(H)[n]$ for any $H$ in $\mathcal{H}$ and any integer $n$.

To prove Theorem 2.5, we are going to verify the conditions of [12, Theorem 2.1.19] for $I$ and $J$. We adopt the notation of [12]: $I$-cof (resp. $J$-cof) is the class of $\mathcal{G}$-cofibrations (resp. the smallest class of maps closed under pushouts, transfinite compositions and retracts which contains $J$ ), and $I$-inj (resp. $J$-inj) is the class of maps which have the right lifting property with respect to $I$ (resp. $J$ ). We will prove that $I$-cof, $J$-cof, $I$-inj and $J$-inj are respectively the class of cofibrations, the class of trivial cofibrations, the class of trivial fibrations and the class of fibrations for this model structure.

Lemma 2.7. For any $E$ in $\mathcal{G}$ and any integer $n$, the complexes $S^{n} E$ and $D^{n} E$ are $\mathcal{G}$-cofibrant. For any $\mathcal{G}$-cofibrant complex $C$, the inclusion $C \oplus C \longrightarrow \operatorname{Cyl}(C)$ is a $\mathcal{G}$ cofibration. Furthermore, if $C$ is acyclic, then this map is also a quasi-isomorphism.

Proof. The pushout square

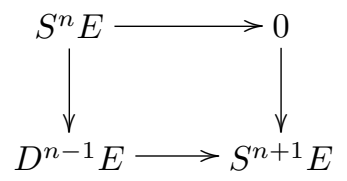

implies the first assertion.

Remember that the cone Cone $(p)$ of a morphism of complexes $p: X \longrightarrow Y$ is defined by Cone $(p)^{n}=Y^{n} \oplus X^{n+1}$, with the differentials given by the formula $d(y, x)$ $=(d y+p x,-d x)$. We then have an obvious short exact sequence

$$
0 \longrightarrow Y \stackrel{u}{\longrightarrow} \operatorname{Cone}(p) \stackrel{v}{\longrightarrow} X[1] \longrightarrow 0,
$$

where $u(y)=(y, 0)$, and $v(y, x)=x$.

Consider an element $E$ of $\mathcal{G}$ and an integer $n$. One checks Cone $\left(1_{S^{n+1} E}\right)=D^{n} E$ and the inclusion of $S^{n+1} E$ in Cone $\left(1_{S^{n+1} E}\right)$ is the canonical inclusion of $S^{n+1} E$ in $D^{n} E$. The complex Cone $\left(1_{D^{n} E}\right)$ is the split acyclic complex

$$
0 \longrightarrow E \longrightarrow E \oplus E \longrightarrow E \longrightarrow 0
$$

where $E \oplus E$ is placed in degree $n+1$. One deduces that the canonical map

$$
D^{n} E \amalg_{S^{n+1} E} \operatorname{Cone}\left(1_{S^{n+1} E}\right) \longrightarrow \operatorname{Cone}\left(1_{D^{n} E}\right)
$$

is a $\mathcal{G}$-cofibration: it corresponds to the obvious inclusion of the complex

$$
0 \longrightarrow 0 \longrightarrow E \oplus E \longrightarrow E \longrightarrow 0
$$

in Cone $\left(1_{D^{n} E}\right)$. In particular, the map $\operatorname{Cone}\left(1_{S^{n+1} E}\right) \longrightarrow \operatorname{Cone}\left(1_{D^{n} E}\right)$ is a $\mathcal{G}$-cofibration. Let $\mathbf{C}$ be the class of maps $A \longrightarrow B$ such that the map

$$
B \amalg_{A} \text { Cone }\left(1_{A}\right) \longrightarrow \operatorname{Cone}\left(1_{B}\right)
$$


induced by the commutative square

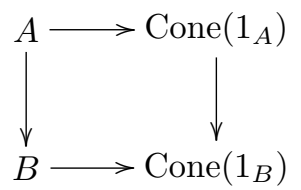

is a $\mathcal{G}$-cofibration. This class of maps is stable by pushouts, transfinite compositions and retracts (the exercise is left to the reader). As $\mathbf{C}$ contains $I$, it has to contain all of $I$-cof. Hence, if $C$ is $\mathcal{G}$-cofibrant, as the map $0 \longrightarrow C$ is in $\mathbf{C}$, then the map $C \longrightarrow$ Cone $\left(1_{C}\right)$ is a $\mathcal{G}$-cofibration. We deduce, for any $\mathcal{G}$-cofibrant complex $C$, that the canonical inclusion $C \longrightarrow$ Cone $\left(1_{C}\right)$ is a $\mathcal{G}$-cofibration. Indeed, we have a pushout square of the following form:

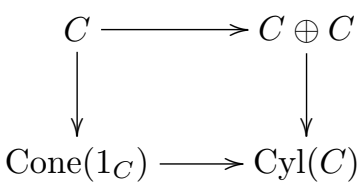

and the $\mathcal{G}$-cofibrations are stable by pushout.

If moreover $C$ is acyclic, then so is $C \oplus C$. As the map $\operatorname{Cyl}(C) \longrightarrow C$ is always a quasi-isomorphism, the complex $\operatorname{Cyl}(C)$ is acyclic as well. Hence the inclusion $C \oplus$ $C \longrightarrow \mathrm{Cyl}(C)$ has to be a quasi-isomorphism.

Lemma 2.8. Any element of $J$-cof is both a $\mathcal{G}$-cofibration and a quasi-isomorphism.

Proof. Any $\mathcal{G}$-cofibration is a monomorphism and the class of maps which are monomorphisms and quasi-isomorphisms is stable by pushouts, transfinite compositions and retracts. Hence the class of $\mathcal{G}$-cofibrations, which are quasi-isomorphisms is also stable by any of these operations. It is then sufficient to check that any map in $J$ is a $\mathcal{G}$-cofibration and a quasi-isomorphism. This follows from Lemma 2.7.

Lemma 2.9. Let $C$ be a complex in $\mathcal{A}$. Then the map $C \longrightarrow 0$ is in $J$-inj if and only if $C$ is $\mathcal{H}$-flasque.

Proof. Let $H$ be in $\mathcal{H}$, and $u$ a map from $H$ to $C[n]$. The data of a chain homotopy between $u$ and the zero map is equivalent to the data of a lifting $k$ in the commutative square below.

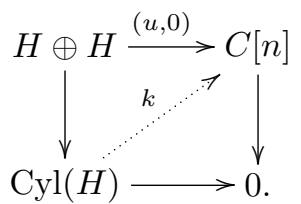

As the right lifting property of $C \longrightarrow 0$ with respect to maps of shape $0 \longrightarrow A$ is always verified, this proves the assertion.

Lemma 2.10. A H-flasque complex $C$ is acyclic if and only if the map $C \longrightarrow 0$ has the right lifting property with respect to $I$. 
Proof. For any complex $C$ in $\mathcal{A}$, as the cohomology group $H^{n}\left(\operatorname{Hom}_{\mathcal{A}}(E, C)\right)$ is the cokernel of the map

$$
\operatorname{Hom}_{\operatorname{Comp}(\mathcal{A})}\left(D^{n-1} E, C\right) \longrightarrow \operatorname{Hom}_{\operatorname{Comp}(\mathcal{A})}\left(S^{n} E, C\right),
$$

it is clear that $C \longrightarrow 0$ is in $I$-inj if and only if the complexes $\operatorname{Hom}_{\mathcal{A}}(E, C)$ are acyclic for any $E$ in $\mathcal{G}$. If $C$ is $\mathcal{H}$-flasque, then for any $E$ in $\mathcal{G}$ and integer $n$, we have the canonical isomorphisms

$$
H^{n}\left(\operatorname{Hom}_{\mathcal{A}}(E, C)\right)=\operatorname{Hom}_{K(\mathcal{A})}(E, C[n])=\operatorname{Hom}_{D(\mathcal{A})}(E, C[n]) .
$$

To finish the proof, it is then sufficient to say that a complex $C$ in $\mathcal{A}$ is acyclic if and only if for any $E$ in $\mathcal{G}$ and integer $n$, the group $\operatorname{Hom}_{D(\mathcal{A})}(E, C[n])$ vanishes.

Lemma 2.11. A map in $J$-inj is a quasi-isomorphism if and only if it is in I-inj.

Proof. Let $p: X \longrightarrow Y$ be map in $J$-inj. We can see that it is an epimorphism: for any element $E$ of $\mathcal{G}$ and any integer $n$, the map

$\operatorname{Hom}_{\mathcal{A}}\left(E, X^{n}\right)=\operatorname{Hom}_{\operatorname{Comp}(\mathcal{A})}\left(D^{n} E, X\right) \longrightarrow \operatorname{Hom}_{\operatorname{Comp}(\mathcal{A})}\left(D^{n} E, Y\right)=\operatorname{Hom}_{\mathcal{A}}\left(E, Y^{n}\right)$

is surjective. As $\mathcal{G}$ is a set of generators of $\mathcal{A}$, this implies that $p$ is an epimorphism. In particular, $\operatorname{ker} p$ is acyclic if and only if $p$ is a quasi-isomorphism. If $p$ is in $I-i n j$, then the map ker $p \longrightarrow 0$ is also in $I$-inj. But $J \subset I$ - $\operatorname{cof}$ (see 2.8), which by, by Lemma 2.9, implies that $\operatorname{ker} p$ is $\mathcal{H}$-flasque. Hence by Lemma 2.10, the complex $\operatorname{ker} p$ is acyclic, and $p$ is a quasi-isomorphism.

Consider now a quasi-isomorphism $p$ in $J$-inj. As $p$ is then an epimorphism, ker $p$ is acyclic, and as right lifting properties are stable by pullback, the map ker $p \longrightarrow 0$ is in $J$-inj. One deduces from 2.9 and 2.10 that the map $\operatorname{ker} p \longrightarrow 0$ is in $I$-inj. Let

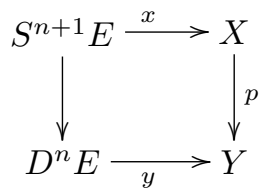

be a commutative square in $\operatorname{Comp}(\mathcal{A})$ where $E$ is in $\mathcal{G}$, and $n$ is an integer. It corresponds to the maps $x: E \longrightarrow X^{n+1}$ and $y: E \longrightarrow Y^{n}$ such that $p x=d y$. To show that the square above admits a lift is equivalent to showing that there is a map $\xi: E \longrightarrow X^{n}$ such that $d \xi=x$ and $p \xi=y$. As $p$ has the right lifting property with respect to $J, \operatorname{Hom}_{\mathcal{A}}\left(E, p^{n}\right)$ is surjective, and so there exists a map $x^{\prime}: E \longrightarrow X^{n}$ such that $p x^{\prime}=y$. We know that $p d x^{\prime}=d p x^{\prime}=d y=p x$, so that $x-d x^{\prime}$ factors through $\operatorname{ker} p$. As the map ker $p \longrightarrow 0$ has the right lifting property with respect to $I$, this implies that there is a map $x^{\prime \prime}: E \longrightarrow X^{n}$ such that $p x^{\prime \prime}=0$ and $d x^{\prime \prime}=x-d x^{\prime}$. We can now set $\xi=x^{\prime}+x^{\prime \prime}$. We have that $p \xi=p x^{\prime}+p x^{\prime \prime}=y$ and $d \xi=d x^{\prime}+d x^{\prime \prime}=x$. Hence the desired lift.

Proof of Theorem 2.5. One deduces from 2.8 and 2.11 that all the conditions of [12, Theorem 2.1.19] are satisfied. In particular, we obtain that we have defined a cofibrantly generated model category with quasi-isomorphisms as weak equivalences and with $I$ (resp. $J$ ) as a set of generators for the cofibrations (resp. for the trivial cofibrations). To see that this structure is cellular, it is sufficient to say that any $\mathcal{G}$-cofibration 
is a monomorphism and that any monomorphism in a Grothendieck category is effective. Properness comes from the fact that cofibrations (resp. fibrations) are monomorphisms (resp. epimorphisms) and standard results of homological algebra (see the end of the proof of 2.1).

It remains to prove the equivalence of conditions (i)-(iii). Using the above definitions and 2.9, it is sufficient to show that (iii) implies (ii). Let $C$ be a $\mathcal{G}$-local complex. One can prove that the full subcategory of $K(\mathcal{A})$ made of $\mathcal{G}$-cofibrant complexes is contained in the localizing subcategory of $K(\mathcal{A})$ generated by $\mathcal{G}$ (in the sense of [19]). Then for any $\mathcal{G}$-cofibrant complex $A$, the map below is an isomorphism.

$$
\operatorname{Hom}_{K(\mathcal{A})}(A, C) \longrightarrow \operatorname{Hom}_{D(\mathcal{A})}(A, C)
$$

In particular, for any $H$ in $\mathcal{H}$ and integer $n$, we have $\operatorname{Hom}_{K(\mathcal{A})}(H, C[n])=0$. Hence $C$ is $\mathcal{H}$-flasque.

Remark 2.12. The model structure of Theorem 2.5 depends only on $\mathcal{G}$ and not on the whole descent structure $(\mathcal{G}, \mathcal{H})$. The data $\mathcal{H}$ is only a tool to get a nice characterization of the fibrant objects in terms of flasque properties (as we will see below, that will be useful to construct total left derived functors). One can prove directly by abstract nonsense that for any Grothendieck category $\mathcal{A}$ with a choice of an essentially small set of generators $\mathcal{G}$, there is a Quillen model category structure on $\operatorname{Comp}(\mathcal{A})$ with quasi-isomorphisms as weak equivalences and $\mathcal{G}$-cofibrations as cofibrations. To prove this, we remark that the class of quasi-isomorphisms is accessible (see the proof of [3, Proposition 3.13]), that any map with the right lifting property with respect to $\mathcal{G}$ cofibrations is a quasi-isomorphism (by virtue of [13, Proposition 1.4]), and apply Jeff Smith's theorem (see [3, Theorem 1.7 and Proposition 1.15]). In fact one can show that any small set $\mathcal{G}$ of generators of $\mathcal{A}$ can be completed in a descent structure for $\mathcal{A}$, in such a way that the description of this model category structure given by 2.5 is always available (but in practice, we essentially always have a canonical choice of descent structure). One can proceed as follows: Choose a set $J_{0}$ of generating trivial cofibration (with respect to the model structure above). Define $\mathcal{H}$ to be the set of the cokernels of the maps in $J_{0}$. Then $(\mathcal{G}, \mathcal{H})$ is a descent structure. Note that the model structure of Theorem 2.5 coincides with the model structure of Christensen and Hovey [5] in the particular case where all the elements of $\mathcal{G}$ are projective. In general, the model structure of Theorem 2.5 is a left Bousfield localization of the model structure associated to the projective class generated by $\mathcal{G}$.

Example 2.13. Consider the notation of 2.4 .

Let $C$ be a complex of sheaves with transfers over $S$.

For any smooth $S$-scheme $X$, we denote by $H^{n} C(X)$ (resp. $\left.H^{n}(X, C)\right)$ the $n$-th cohomology of the complex of abelian groups made by the sections of $C$ over $X$ (resp. the $n$-th Nisnevich hypercohomology of $X$ with coefficients in the complex $C$ where we forget transfers).

We will say that $C$ is Nisnevich fibrant if it is fibrant with respect to the descent structure $\left(\mathcal{G}_{S}, \mathcal{H}_{S}\right)$. According to the previous theorem and [8, 4.2.9], this means one of the following equivalent conditions are true: 
(i) For any smooth $S$-scheme $X$ and any integer $n$, the canonical map

$$
H^{n}(C(X)) \rightarrow H_{\mathrm{Nis}}^{n}(X ; C)
$$

is an isomorphism.

(ii) For any smooth $S$-scheme $X$, any Nisnevich hypercovering $\mathcal{X}$ of $X$, and any integer $n$, the canonical map $H^{n}(C(X)) \rightarrow H^{n}(\operatorname{Tot} C(\mathcal{X}))$ is an isomorphism.

\section{4 .}

Let $\mathcal{A}$ and $\mathcal{A}^{\prime}$ be two Grothendieck categories. Suppose that $(\mathcal{G}, \mathcal{H})\left(\operatorname{resp} .\left(\mathcal{G}^{\prime}, \mathcal{H}^{\prime}\right)\right)$ is a descent structure on $\mathcal{A}$ (resp. $\mathcal{A}^{\prime}$ ). A functor $f^{*}: \mathcal{A}^{\prime} \longrightarrow \mathcal{A}$ satisfies descent (with respect to the above descent structures) if it satisfies the following conditions:

(i) The functor $f^{*}$ preserves colimits.

(ii) For any $E^{\prime}$ in $\mathcal{G}^{\prime}, f^{*}\left(E^{\prime}\right)$ is a direct sum of elements of $\mathcal{G}$.

(iii) For any $H^{\prime}$ in $\mathcal{H}^{\prime}, f^{*}\left(H^{\prime}\right)$ is in $\mathcal{H}$.

It is a standard fact that a functor between Grothendieck categories preserves colimits if and only if it has a right adjoint. Note also that any functor which preserves colimits preserves, in particular, finite direct sums, so that any functor which satisfies descent is additive.

Theorem 2.14. Under the assumptions above, let $f^{*}: \mathcal{A}^{\prime} \longrightarrow \mathcal{A}$ be a functor that preserves colimits, and $f_{*}: \mathcal{A} \longrightarrow \mathcal{A}^{\prime}$ be its right adjoint. If $f^{*}$ satisfies descent, then the pair of adjoint functors

$$
f^{*}: \operatorname{Comp}\left(\mathcal{A}^{\prime}\right) \longrightarrow \operatorname{Comp}(\mathcal{A}) \quad \text { and } \quad f_{*}: \operatorname{Comp}(\mathcal{A}) \longrightarrow \operatorname{Comp}\left(\mathcal{A}^{\prime}\right)
$$

is a Quillen pair with respect to the model structures associated to $\mathcal{G}$ and $\mathcal{G}^{\prime}$ by Theorem 2.5. In particular, the functors $f^{*}$ and $f_{*}$ have the functors

$$
\mathbf{L} f^{*}: D\left(\mathcal{A}^{\prime}\right) \longrightarrow D(\mathcal{A}) \text { and } \mathbf{R} f_{*}: D(\mathcal{A}) \longrightarrow D\left(\mathcal{A}^{\prime}\right)
$$

as left and right derived functors respectively, and $\mathbf{L} f^{*}$ is left adjoint to $\mathbf{R} f_{*}$.

Proof. If $f^{*}$ satisfies descent, then one checks easily that it preserves cofibrations; see [12, Lemma 2.1.20]. It is then sufficient to show that it sends trivial cofibrations to quasi-isomorphisms. As $f^{*}$ preserves colimits and cofibrations, by virtue of loc. cit, it is sufficient to prove that any element of $f^{*}\left(J^{\prime}\right)$ is a quasi-isomorphism, where $J^{\prime}$ is the set of trivial cofibrations defined as in 2.3. This comes directly from the fact that $f^{*}\left(\mathcal{H}^{\prime}\right)$ is contained in $\mathcal{H}$ and that $f^{*}$ commutes to the formation of cones and cylinders (just because it is additive).

Remark 2.15. Consider three Grothendieck categories endowed with descent structures $\mathcal{A}, \mathcal{A}^{\prime}$, and $\mathcal{A}^{\prime \prime}$. Let $f^{\prime *}: \mathcal{A}^{\prime \prime} \longrightarrow \mathcal{A}^{\prime}$ and $f^{*}: \mathcal{A}^{\prime} \longrightarrow \mathcal{A}$ be two functors commuting to colimits and satisfying descent, and denote by $f_{*}^{\prime}$ and $f_{*}$ their corresponding right adjoints. Then it follows easily from general abstract nonsense about Quillen adjunctions and the preceding theorem that we have canonical isomorphisms of total derived functors

$$
\mathbf{L} f^{*} \circ \mathbf{L} f^{\prime *} \simeq \mathbf{L}\left(f^{*} \circ f^{\prime *}\right) \quad \text { and } \quad \mathbf{R}\left(f_{*}^{\prime} \circ f_{*}\right) \simeq \mathbf{R} f_{*}^{\prime} \circ \mathbf{R} f_{*} .
$$


Example 2.16. Consider the notation of Example 2.4.

Let $f: T \longrightarrow S$ be a morphism between noetherian regular schemes. In $[8,4.2 .5]$, the base change functor $f^{*}: \mathcal{N}_{S}^{t r} \longrightarrow \mathcal{N}_{T}^{t r}$ is defined together with its right adjoint $f_{*}: \mathcal{N}_{T}^{t r} \longrightarrow \mathcal{N}_{S}^{t r}$. By definition, $f^{*} L_{S}(X)=L_{T}\left(X \times_{S} T\right)$ for any smooth finite type $S$-scheme $X$. Thus $f^{*}$ obviously satisfies descent with respect to the descent structures $\left(\mathcal{G}_{S}, \mathcal{H}_{S}\right)$ and $\left(\mathcal{G}_{T}, \mathcal{H}_{T}\right)$ defined in 2.4. Thus we get a pair of adjoint derived functors

$$
\mathbf{L} f^{*}: D\left(\mathcal{N}_{S}^{t r}\right) \longrightarrow D\left(\mathcal{N}_{T}^{t r}\right) \text { and } \mathbf{R} f_{*}: D\left(\mathcal{N}_{T}^{t r}\right) \longrightarrow D\left(\mathcal{N}_{S}^{t r}\right) .
$$

Suppose moreover that $f: T \rightarrow S$ is smooth of finite type. Then, in $[8,4.2 .5]$, the functor $f_{\sharp}: \mathcal{N}_{T}^{t r} \rightarrow \mathcal{N}_{S}^{t r}$ is defined, which forgets the base: for any smooth finite type $T$-scheme $Y \stackrel{\pi}{\rightarrow} T, f_{\sharp} L_{T}(Y)=L_{S}(Y \stackrel{\pi}{\rightarrow} T \stackrel{f}{\rightarrow} S)$. It is proved in loc. cit. that this functor is left adjoint to the functor $f^{*}$. Obviously, $f_{\sharp}$ satisfies descent, so that we get a pair of adjoint derived functors

$$
\mathbf{L} f_{\sharp}: D\left(\mathcal{N}_{S}^{t r}\right) \longrightarrow D\left(\mathcal{N}_{T}^{t r}\right) \text { and } \quad \mathbf{R} f^{*}=f^{*}: D\left(\mathcal{N}_{T}^{t r}\right) \longrightarrow D\left(\mathcal{N}_{S}^{t r}\right) .
$$

\section{Derived tensor product}

\section{1.}

Let $\mathcal{A}$ be a Grothendieck category endowed with a closed symmetric monoidal structure $^{2}$ An object $X$ of $\mathcal{A}$ will be said to be flat if the functor defined by the tensor product with $X$ is exact. An essentially small family of generators $\mathcal{G}$ on $\mathcal{A}$ will be said to be flat if the following conditions are satisfied:

(i) Any element of $\mathcal{G}$ is flat.

(ii) The set $\mathcal{G}$ is stable by tensor product up to isomorphism and the unit object of $\mathcal{A}$ is in $\mathcal{G}$.

The category $\operatorname{Comp}(\mathcal{A})$ of complexes in $\mathcal{A}$ is canonically endowed with a symmetric monoidal structure induced by the one of $\mathcal{A}$ by setting for two complexes $X$ and $Y$

$$
(X \otimes Y)^{n}=\bigoplus_{p+q=n} X^{p} \otimes Y^{q} .
$$

The differential of $X \otimes Y$ is given by the Leibniz formula

$$
d(x \otimes y)=d x \otimes y+(-1)^{p} x \otimes d y,
$$

where $p$ is the degree of $x$. The unit is just the unit of $\mathcal{A}$ concentrated in degree zero. The associativity structure comes from the one on $\mathcal{A}$, and the symmetry is induced by the one on $\mathcal{A}$ with the usual sign convention

$$
\begin{aligned}
X \otimes Y & \longrightarrow Y \otimes X, \\
x \otimes y & \longmapsto(-1)^{p q} y \otimes x,
\end{aligned}
$$

where $p$ and $q$ are the degree of $x$ and $y$ respectively. A descent structure $(\mathcal{G}, \mathcal{H})$ on $\mathcal{A}$ is weakly flat if the following conditions are satisfied:

\footnotetext{
${ }^{2}$ Remember that a closed symmetric monoidal category is a symmetric monoidal category with
} internal Hom's. 
(i) The set $\mathcal{G}$ is stable by tensor product up to isomorphism and the unit object of $\mathcal{A}$ is in $\mathcal{G}$.

(ii) For any $E$ in $\mathcal{G}$ and any $H$ in $\mathcal{H}$, the complex $E \otimes H$ is acyclic.

A descent structure $(\mathcal{G}, \mathcal{H})$ on $\mathcal{A}$ is flat if it is weakly flat, and if for any complex $C$ on $\mathcal{A}$ and any $H$ in $\mathcal{H}$, the complex $C \otimes H$ is acyclic. We will see that a descent structure is flat if and only if the underlying generating family is flat; see Proposition 3.7 below.

Example 3.1. Consider a Grothendieck site $\mathcal{S}$ and a sheaf of rings $\mathcal{R}$ on $\mathcal{S}$. Consider the usual tensor product on the category of sheaves of $\mathcal{R}$-modules. If the category $\mathcal{S}$ has finite products, then the descent structure of Example 2.3 is flat. This comes from the fact that for two objects $X$ and $Y$ in $\mathcal{S}$, we have the formula $\mathcal{R}(X \times Y)=$ $\mathcal{R}(X) \otimes_{\mathcal{R}} \mathcal{R}(Y)$ and from the fact that the free sheaves of shape $\mathcal{R}(X)$ are flat.

Similarly, given a quasi-separated and quasi-compact scheme $X$, the natural descent structure on the category of quasi-coherent $\mathcal{O}_{X}$-modules is flat.

Proposition 3.2. Let $(\mathcal{G}, \mathcal{H})$ be a weakly flat descent structure on $\mathcal{A}$. The corresponding $\mathcal{G}$-model structure on $\operatorname{Comp}(\mathcal{A})$ is a symmetric monoidal model category (see [12]). In particular, the tensor product on $\operatorname{Comp}(\mathcal{A})$ has a total left derived functor

$$
\begin{aligned}
D(\mathcal{A}) \times D(\mathcal{A}) & \longrightarrow D(\mathcal{A}), \\
(A, B) & \longmapsto A \otimes^{\mathbf{L}} B .
\end{aligned}
$$

Proof. First of all, consider two $\mathcal{G}$-cofibrations $a: A \longrightarrow A^{\prime}$ and $b: B \longrightarrow B^{\prime}$. Then the map

$$
c: A \otimes B^{\prime} \amalg_{A \otimes B} A^{\prime} \otimes B \longrightarrow A^{\prime} \otimes B^{\prime}
$$

induced by the commutative square

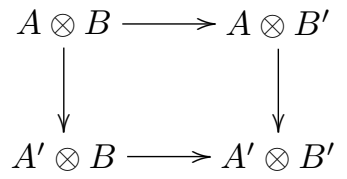

is a $\mathcal{G}$-cofibration: according to [12, Lemma 4.2.4], it is sufficient to check this property when $a$ and $b$ are in $I$, but in this case, this follows easily from condition (i) of the definition of weak flatness. Furthermore, if $b$ is a quasi-isomorphism, then so is $c$ : by loc. cit., it is sufficient to prove this assuming that $a$ is in $I$ and $b$ is in $J$, in which case this is trivial using condition (ii). This achieves the proof.

Example 3.3. Consider the notation of Example 2.4.

The category $\mathcal{N}_{S}^{t r}$ admits a closed symmetric monoidal structure from $[8,4.2 .12$, 4.12.14]. This structure satisfies the fundamental property that $L_{S}(X) \otimes L_{S}(Y)=$ $L_{S}\left(X \times_{S} Y\right)$. Thus, the descent structure $\left(\mathcal{G}_{S}, \mathcal{H}_{S}\right)$ is obviously weakly flat and we get a left derived tensor product $\otimes^{\mathbf{L}}$ on $D\left(\mathcal{N}_{S}^{t r}\right)$.

However, we do not know if $\left(\mathcal{G}_{S}, \mathcal{H}_{S}\right)$ is flat, which is actually why we also consider the weakly flat condition.

Proposition 3.4. Let $(\mathcal{G}, \mathcal{H})$ be a weakly flat descent structure on a closed symmetric monoidal Grothendieck category, and let $C$ be a complex in $\mathcal{A}$. We assume the following assumptions: 
(a) For any $H$ in $\mathcal{H}$, and for any integer $n$, the complex $H \otimes C^{n}$ is acyclic.

(b) For any $H$ in $\mathcal{H}$, and for any integer $n$, the complex $H \otimes \operatorname{ker}\left(C^{n} \longrightarrow C^{n+1}\right)$ is acyclic.

Then the functor $A \longmapsto A \otimes C$ is a left Quillen functor from the $\mathcal{G}$-model structure on $\operatorname{Comp}(\mathcal{A})$ to the injective model structure on $\operatorname{Comp}(\mathcal{A})$. In particular, for any complex $C$ over $\mathcal{A}$ and any quasi-isomorphism which is also a $\mathcal{G}$-cofibration $X \longrightarrow Y$, the map $C \otimes X \longrightarrow C \otimes Y$ is a quasi-isomorphism and a monomorphism.

Proof. Given two integers $p<q$, write $C_{p, q}$ for the complex obtained from $C$ by the formula

$$
C_{p, q}^{i}= \begin{cases}0 & \text { if } i \leqslant p \text { or } i>q, \\ C^{i} & \text { if } p<i<q, \\ \operatorname{ker}\left(C^{q} \longrightarrow C^{q+1}\right) & \text { if } i=q .\end{cases}
$$

The complex $C$ is a filtered colimit of the complexes $C_{p, q}$. These complexes are bounded, and for $p+1<q$, we have an obvious degreewise split short exact sequence of complexes

$$
0 \longrightarrow C_{p+1, q} \longrightarrow C_{p, q} \longrightarrow S^{p}\left(C^{p}\right) \longrightarrow 0 .
$$

This implies that, for any complex $K$, we have a short exact sequence

$$
0 \longrightarrow K \otimes C_{p+1, q} \longrightarrow K \otimes C_{p, q} \longrightarrow K \otimes S^{p}\left(C^{p}\right) \longrightarrow 0
$$

(it is sufficient to check this for bounded complexes $K$ ). Our assumptions thus imply that $H \otimes C_{p, q}$ is acyclic for any $p<q$. In conclusion, $H \otimes C$ is the filtered colimit of the acyclic complexes $H \otimes C_{p, q}$, hence it is acyclic.

To prove that the tensor product by $C$ defines a left Quillen functor, by applying [12, Lemma 2.1.20], it is sufficient to prove that for any map $f$ in $I$ (resp. in $J$ ), the map $f \otimes C$ is a monomorphism (resp. a quasi-isomorphism). The only nontrivial part to check is that for any $H$ in $\mathcal{H}$, the map

$$
H \otimes C \oplus H \otimes C \longrightarrow \operatorname{Cyl}(H) \otimes C
$$

is a quasi-isomorphism, or in other words, that $\mathrm{Cyl}(H) \otimes C$ is acyclic. But we have a push-out square

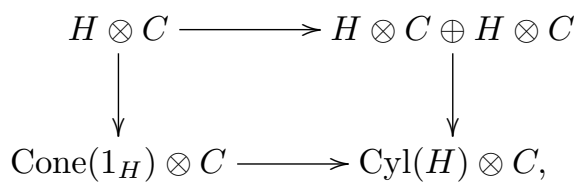

where the left vertical map is a monomorphism. As we already know that $H \otimes C$ is acyclic, it is sufficient to check that $\operatorname{Cone}\left(1_{H}\right) \otimes C$ is acyclic. For this, it is sufficient to check that, for any $p<q$, the complex Cone $\left(1_{H}\right) \otimes C_{p, q}$ is acyclic. If $C_{p, q}$ is concentrated in one degree, then we have Cone $\left(1_{H}\right) \otimes C_{p, q}=\operatorname{Cone}\left(1_{H \otimes C_{p, q}}\right)$, and this is trivial. For any integer $i$, we also have acyclic complexes: $\operatorname{Cone}\left(1_{H}\right) \otimes C^{i}=\operatorname{Cone}\left(1_{H \otimes C^{i}}\right)$. The acyclicity of Cone $\left(1_{H}\right) \otimes C_{p, q}$ now follows by an easy induction. 
Corollary 3.5. Let $\mathcal{A}$ be a closed symmetric monoidal Grothendieck category endowed with a flat descent structure $(\mathcal{G}, \mathcal{H})$. Then the associated $\mathcal{G}$-model structure is a monoidal model category satisfying the monoid axiom ${ }^{3}$ (see [20]).

Proof. The first assertion follows from Proposition 3.2. As the trivial cofibrations of the injective model structure on $\operatorname{Comp}(\mathcal{A})$ are, in particular, stable by pushouts and transfinite compositions, the monoid axiom follows from Proposition 3.4.

Lemma 3.6. Let $\mathcal{A}$ be a closed symmetric monoidal Grothendieck category, and $\mathcal{G}$ be an essentially small set of generators of $\mathcal{A}$. Consider a map of complexes $i: A \longrightarrow B$ such that for any $E$ in $\mathcal{G}, i \otimes E$ is a quasi-isomorphism. Then for any $\mathcal{G}$-cofibrant complex $C$, the map of complexes $i \otimes C$ is a quasi-isomorphism.

Proof. One easily checks that the map of complexes $i \otimes S^{n} E$ and $i \otimes D^{n} E$ are quasiisomorphisms for any $E$ in $\mathcal{G}$ and any integer $n$. If $C^{\prime}$ is a direct summand of $C$, and if $i \otimes C$ is a quasi-isomorphism, then so is $i \otimes C^{\prime}$. If

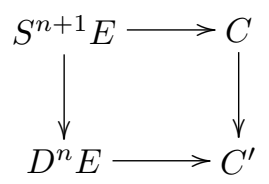

is a pushout in $\operatorname{Comp}(\mathcal{A})$ with $E$ in $\mathcal{G}$ and $n$ an integer, then if $i \otimes C$ is acyclic, so is $i \otimes C^{\prime}$. This comes from the cube lemma applied to the injective model structure (see [12, Lemma 5.2.6]), and the facts that the induced map $A \otimes S^{n} E \longrightarrow A \otimes D^{n} E$ is a monomorphism for any $A$, and that the tensor product by any complex preserves pushouts (remember it has a right adjoint). We know by the small object argument applied to $I$ that any $\mathcal{G}$-cofibrant complex $C$ is a direct summand of a well-ordered colimit of complexes constructed inductively by some pushout of the type above. As quasi-isomorphisms are stable by filtered colimits in a Grothendieck category, one deduces that for any $\mathcal{G}$-cofibrant complex $C, i \otimes C$ is a quasi-isomorphism.

Proposition 3.7. Let $\mathcal{A}$ be a closed symmetric monoidal Grothendieck category, and $(\mathcal{G}, \mathcal{H})$ a descent structure on $\mathcal{A}$. If $\mathcal{G}$ is flat, then the descent structure $(\mathcal{G}, \mathcal{H})$ is flat. Moreover, the tensor product by any $\mathcal{G}$-cofibrant complex preserves quasiisomorphisms.

Proof. Suppose that $\mathcal{G}$ is flat. It is then clear that the descent structure is weakly flat. In particular, the tensor product on $\operatorname{Comp}(\mathcal{A})$ has a left derived functor defined using the $\mathcal{G}$-model structure (3.2). Let $C$ be a $\mathcal{G}$-cofibrant complex. By Lemma 3.6, we can see that the functor $X \longmapsto C \otimes X$ preserves quasi-isomorphisms, so that for any complex $X$ over $\mathcal{A}$, the canonical map $C \otimes^{\mathbf{L}} X \longrightarrow C \otimes X$ is an isomorphism in $D(\mathcal{A})$. One concludes that for any quasi-isomorphism between $\mathcal{G}$-cofibrant complexes $C \longrightarrow C^{\prime}$ and any complex $X$, the map $C \otimes X \longrightarrow C^{\prime} \otimes X$ is a quasi-isomorphism. In particular, for any $H$ in $\mathcal{H}$ and any complex $X, H \otimes X$ is acyclic and the descent structure $(\mathcal{G}, \mathcal{H})$ is flat.

\footnotetext{
${ }^{3}$ The monoid axiom is a technical property that makes life easier when one wants to define the homotopy theory of monoids or of modules over a given monoid in a symmetric monoidal model category. In our particular case, this can be seen as the second statement in Proposition 3.4.
} 
Remark 3.8. If $\mathcal{G}$ is a flat generating family of $\mathcal{A}$, then for any complex $X$, the total left derived functor of $Y \longmapsto X \otimes Y$ (cf. 3.4) is isomorphic to the functor $Y \longmapsto X \otimes \mathbf{L} Y$ (obtained as the total left derived functor of the functor $(A, B) \longmapsto A \otimes B$; cf. 3.2). This is an immediate consequence of the fact that the tensor product by a $\mathcal{G}$-cofibrant complex preserves quasi-isomorphisms. This property is of course interesting by itself, but it will also be used in a very essential way to prove the monoid axiom after further localizations (see e.g. Corollary 4.11).

\section{2 .}

Consider a closed symmetric monoidal Grothendieck category $\mathcal{A}$ endowed with a weakly flat descent structure $(\mathcal{G}, \mathcal{H})$. We will now consider a way to enlarge this descent structure in a way which preserves weak flatness.

Let $T$ be a given object of $\mathcal{A}$, define $\mathcal{G}[T]$ to be the family of objects of the form $E \otimes T^{\otimes n}$ with $E$ in $\mathcal{G}$ and $n \geqslant 0$.

We will say that $T$ is weakly flat (with respect to $(\mathcal{G}, \mathcal{H})$ ) if it has the two following properties:

(a) For any $H$ in $\mathcal{H}$ and any integer $n \geqslant 0$, the complex $H \otimes T^{\otimes n}$ is acyclic.

(b) For any object $F$ in $\mathcal{G}[T]$, there exists a quasi-isomorphism $H \longrightarrow F$, with $H$ a $\mathcal{G}$-cofibrant complex, such that for any object $F^{\prime}$ in $\mathcal{G}[T]$, the map $H \otimes F^{\prime} \longrightarrow$ $F \otimes F^{\prime}$ is a quasi-isomorphism.

A quasi-isomorphism $H \longrightarrow F$ satisfying the property described in (b) will be called a weakly flat resolution of $F$.

Suppose that $T$ is weakly flat. For each object $F$ in $\mathcal{G}[T]$, choose a weakly flat resolution $u_{F}: H_{F} \longrightarrow F$ satisfying condition (b) above. Define $\mathcal{H}[T]$ to be $\mathcal{H} \cup \mathcal{H}^{\prime}$, where $\mathcal{H}^{\prime}$ is the set of complexes of shape Cone $\left(u_{F}\right)$.

Proposition 3.9. The couple $(\mathcal{G}[T], \mathcal{H}[T])$ is a weakly flat descent structure.

Proof. Note that the elements of $\mathcal{H}[T]$ are $\mathcal{G}[T]$-cofibrant and acyclic. To prove we have defined a descent structure, it remains to prove that any $\mathcal{H}[T]$-flasque complex is $\mathcal{G}[T]$-local. Let $C$ be an $\mathcal{H}[T]$-flasque complex. It is, in particular, $\mathcal{H}$-flasque, so that for any $\mathcal{G}$-cofibrant complex $H$ (e.g. $H=H_{F}$ ), we have

$$
\operatorname{Hom}_{K(\mathcal{A})}(H, C)=\operatorname{Hom}_{D(\mathcal{A})}(H, C) .
$$

We also have distinguished triangles

$$
H_{F} \longrightarrow F \longrightarrow \text { Cone }\left(u_{F}\right) \longrightarrow H_{F}[1]
$$

in the homotopy category $K(\mathcal{A})$, so that we deduce that $C$ is $\mathcal{G}[T]$-local.

To prove the weak flatness, we first notice that condition (a) implies that for any $n \geqslant 0$, the functor $C \longmapsto C \otimes T^{\otimes n}$ is a left Quillen functor from the $\mathcal{G}$-model structure to the injective model structure. Condition (b) completes the proof.

Example 3.10. Let $T$ be an object of $\mathcal{A}$. Suppose that for any integer $n \geqslant 0$, there 
exists a short exact sequence of the form

$$
0 \longrightarrow A_{n} \longrightarrow B_{n} \longrightarrow T^{\otimes n} \longrightarrow 0,
$$

with $A_{n}$ and $B_{n}$ in $\mathcal{G}$, and such that for any object $F$ in $\mathcal{G}[T]$, the sequence

$$
0 \longrightarrow A_{n} \otimes F \longrightarrow B_{n} \otimes F \longrightarrow T^{\otimes n} \otimes F \longrightarrow 0
$$

remains exact. Then $T$ is weakly flat.

Condition (a) is easy to check: if $H$ is a $\mathcal{G}$-cofibrant complex, then it is degreewise a direct factor of a sum of elements of $\mathcal{G}$, so that we get short exact sequences

$$
0 \longrightarrow A_{n} \otimes H \longrightarrow B_{n} \otimes H \longrightarrow T^{\otimes n} \otimes H \longrightarrow 0 .
$$

If moreover $H$ is acyclic, as the complexes $A_{n} \otimes H$ and $B_{n} \otimes H$ are then acyclic, then the complex $T^{\otimes n} \otimes H$ is acyclic as well. It remains to verify condition (b). For an object $E$ in $\mathcal{G}$ and an integer $n$, define $H$ to be the complex

$$
H=\operatorname{Cone}\left(A_{n} \otimes E \longrightarrow B_{n} \otimes E\right) .
$$

The obvious quasi-isomorphism $H \longrightarrow T^{\otimes n} \otimes E$ is a weakly flat resolution of $F=$ $T^{\otimes n} \otimes E$.

\section{Localization of derived categories}

\section{1.}

From now on, we consider an abelian Grothendieck category $\mathcal{A}$ endowed with a descent structure $(\mathcal{G}, \mathcal{H})$.

Let $\mathcal{T}$ be an essentially small set of complexes over $\mathcal{A}$. For any $A$ in $\mathcal{T}$, we choose a quasi-isomorphism $T^{\prime} \longrightarrow T$ with $T^{\prime} \mathcal{G}$-cofibrant (this exists using the cofibrant resolutions of the $\mathcal{G}$-model structure on $\mathcal{A}$ ), and set

$$
\mathcal{T}^{\prime}=\left\{T^{\prime} \mid T \in \mathcal{T}\right\}
$$

A morphism of complexes $X \rightarrow Y$ over $\mathcal{A}$ is a $\mathcal{T}$-equivalence if, for any $\mathcal{H} \cup \mathcal{T}^{\prime}$-flasque complex $K$, the map

$$
\operatorname{Hom}_{D(\mathcal{A})}(Y, K) \longrightarrow \operatorname{Hom}_{D(\mathcal{A})}(X, K)
$$

is bijective.

Lemma 4.1. Let $K$ be complex over $\mathcal{A}$. The following conditions are equivalent:

(i) The complex $K$ is $\mathcal{H} \cup \mathcal{T}^{\prime}$-flasque.

(ii) The complex $K$ is $\mathcal{G}$-local, and for any $T$ in $\mathcal{T}$ and any integer $n$, the group $\operatorname{Hom}_{D(\mathcal{A})}(T, K[n])$ vanishes.

(iii) The complex $K$ is $\mathcal{G}$-local, and for any $\mathcal{T}$-equivalence $X \longrightarrow Y$, the map

$$
\operatorname{Hom}_{D(\mathcal{A})}(Y, K) \longrightarrow \operatorname{Hom}_{D(\mathcal{A})}(X, K)
$$

is bijective.

Proof. We know that $K$ is $\mathcal{H}$-flasque if and only if it is $\mathcal{G}$-local, and it is clear that $K$ is $\mathcal{H} \cup \mathcal{T}^{\prime}$-flasque if and only if it is $\mathcal{H}$-flasque and $\mathcal{T}^{\prime}$-flasque. Moreover, if $K$ is 
$\mathcal{G}$-local, for any quasi-isomorphism $T^{\prime} \longrightarrow T$ with $T^{\prime} \mathcal{G}$-cofibrant, then the map

$$
\operatorname{Hom}_{D(\mathcal{A})}(T, K) \longrightarrow \operatorname{Hom}_{K(\mathcal{A})}\left(T^{\prime}, K\right)=\operatorname{Hom}_{D(\mathcal{A})}\left(T^{\prime}, K\right)
$$

is bijective. This implies that conditions (i) and (ii) are equivalent. As (i) implies (iii) by definition, it remains to show that (iii) implies (i). But this comes from the obvious fact that for any $T^{\prime}$ in $\mathcal{T}^{\prime}$, the map $T^{\prime} \longrightarrow 0$ is a $\mathcal{T}$-equivalence.

Proposition 4.2. Let $K$ be a complex over $\mathcal{A}$. The following conditions are equivalent:

(i) For any $T$ in $\mathcal{T}$, and any integer $n$, the group $\operatorname{Hom}_{D(\mathcal{A})}(T, K[n])$ vanishes.

(ii) For any $\mathcal{T}$-equivalence $X \longrightarrow Y$, the map

$$
\operatorname{Hom}_{D(\mathcal{A})}(Y, K) \longrightarrow \operatorname{Hom}_{D(\mathcal{A})}(X, K)
$$

is bijective.

Proof. Let $K \longrightarrow K^{\prime}$ be a quasi-isomorphism with $K^{\prime} \mathcal{G}$-local - this exists using the fibrant resolutions of the $\mathcal{G}$-model structure. It is clear that condition (i) (resp. (ii)) is verified for $K$ if and only if it is for $K^{\prime}$; thus we can assume that $K$ is $\mathcal{G}$-local. This proposition then follows immediately from Lemma 4.1.

\section{2 .}

We will say that a complex $K$ is $\mathcal{T}$-local if it satisfies one of the equivalent conditions of the proposition above. It is straightforward to check that a map of complexes $X \longrightarrow Y$ is a $\mathcal{T}$-equivalence if and only if for any $\mathcal{T}$-local complex $K$, the map $\operatorname{Hom}_{D(\mathcal{A})}(Y, K) \longrightarrow \operatorname{Hom}_{D(\mathcal{A})}(X, K)$ is bijective. Hence the notion of $\mathcal{T}$-equivalence does not depend on the descent structure $(\mathcal{G}, \mathcal{H})$.

Proposition 4.3. The category $\operatorname{Comp}(\mathcal{A})$ admits a proper cellular model category structure with $\mathcal{T}$-equivalences as weak equivalences and $\mathcal{G}$-cofibrations as cofibrations. This model category structure on $\operatorname{Comp}(\mathcal{A})$ is the left Bousfield localization of the $\mathcal{G}$-model structure by the maps $0 \longrightarrow T[n]$ for $T$ in $\mathcal{T}$ and $n$ in $\mathbb{Z}$. We will call this model category structure the $\mathcal{G}$-model structure associated to $\mathcal{T}$.

The fibrant objects for this model structure can be characterized as the $\mathcal{G}$-local and $\mathcal{T}$-local complexes.

Let $D_{\mathcal{T}}(\mathcal{A})$ denote the localization of $\operatorname{Comp}(\mathcal{A})$ by the $\mathcal{T}$-equivalences. If $\mathrm{T}(\mathcal{A})$ is the localizing subcategory of $D(\mathcal{A})$ generated by the objects $T[n]$ for $T$ in $\mathcal{T}$ and any integer $n^{4}$, then $D_{\mathcal{T}}(\mathcal{A})=D(\mathcal{A}) / \mathrm{T}(\mathcal{A})$. In particular, the category $D_{\mathcal{T}}(\mathcal{A})$ is triangulated. Moreover, the localization functor

$$
D(\mathcal{A}) \longrightarrow D_{\mathcal{T}}(\mathcal{A})
$$

is triangulated, and it has a right adjoint that is fully faithful and identifies $D_{\mathcal{T}}(\mathcal{A})$ with the full subcategory of $D(\mathcal{A})$ that consists of $\mathcal{T}$-local complexes.

Remark 4.4. It is possible to give an explicit description of the fibrations for this model category structure; see Corollary 5.6.

${ }^{4}$ That is, the smallest full triangulated subcategory of $D(\mathcal{A})$ stable by direct sums which contains $\mathcal{T}$. 
Proof. As the $\mathcal{G}$-model structure is (left) proper and cellular, we can apply Hirschhorn's general construction [11] and obtain a cellular left proper model category structure on $\operatorname{Comp}(\mathcal{A})$ as the left Bousfield localization of the $\mathcal{G}$-model structure by the maps $0 \longrightarrow T[n]$ for $T$ in $\mathcal{T}$ and $n$ in $\mathbb{Z}$. By definition, the cofibrations of this latter model structure still are the $\mathcal{G}$-cofibrations. There remains to show three points to conclude: characterize the fibrant objects as announced in the statement of this proposition, identify $D_{\mathcal{T}}(\mathcal{A})$ with a full subcategory of $D(\mathcal{A})$, and prove that this model category structure is right proper. Let us show the first point. For any pair $(X, Y)$ of objects of $\operatorname{Comp}(\mathcal{A})$, we can define functorially (at least in the homotopy category of simplicial sets) a pointed simplicial set $\mathbf{R H o m}(X, Y)$ whose higher homotopy groups are the $\operatorname{Hom}_{D(\mathcal{A})}(X[n], Y)$ 's for $n \geqslant 0$. In Hirschhorn's construction, the fibrant objects of the Bousfield localization of the $\mathcal{G}$-model structure are then characterized as the objects $K$ such that for any integer $n$,

$$
\mathbf{R H o m}(T[n], K) \longrightarrow \mathbf{R H o m}(0[n], K) \simeq 0
$$

is a (weak) homotopy equivalence. But this is is clearly equivalent to saying that the groups $\operatorname{Hom}_{D(\mathcal{A})}(T, K[n])$ vanish. The general theory of left Bousfield localization shows that the localization functor $D(\mathcal{A}) \longrightarrow D_{\mathcal{T}}(\mathcal{A})$ has a fully faithful right adjoint whose essential image is made of fibrant objects, that are exactly the $\mathcal{G}$ local $\mathcal{T}^{\prime}$-flasque complexes. To identify $D_{\mathcal{T}}(\mathcal{A})$ with the quotient $D(\mathcal{A}) / \mathrm{T}(\mathcal{A})$, by Proposition 4.2 and the theory of left Bousfield localization of triangulated categories (see [19]), it is sufficient to prove that for any $C$ in $\mathrm{T}(A)$, the map $0 \longrightarrow C$ is a $\mathcal{T}$ equivalence. This is an easy exercise left to the reader. To prove right properness, consider now a pullback square of complexes

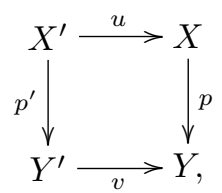

where $p$ is a fibration for the localized model structure, and $v$ is a $\mathcal{T}$-equivalence. We have to prove that $u$ is a $\mathcal{T}$-equivalence. But $p$ is also a fibration for the $\mathcal{G}$-model structure, and as the $\mathcal{G}$-model structure is right proper, this square is homotopy cartesian with respect to the $\mathcal{G}$-model structure. This is equivalent to saying that we have a canonical distinguished triangle

$$
X^{\prime} \longrightarrow Y^{\prime} \oplus X \longrightarrow Y \longrightarrow X^{\prime}[1]
$$

in $D(\mathcal{A})$. As the localization functor from $D(\mathcal{A})$ to $D_{\mathcal{T}}(\mathcal{A})$ is triangulated, this is also a distinguished triangle in $D_{\mathcal{T}}(\mathcal{A})$. But $v$ is an isomorphism in $D_{\mathcal{T}}(\mathcal{A})$, which then implies that $u$ is also an isomorphism. As a morphism in a model category is a weak equivalence if and only if it is an isomorphism in the corresponding homotopy category, this implies that $u$ is a $\mathcal{T}$-equivalence. 
Proposition 4.5. A pushout square of complexes of $\mathcal{A}$,

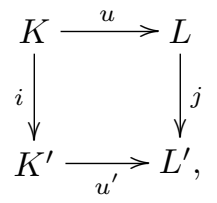

in which $i$ is a monomorphism, is a homotopy pushout square with respect to the $\mathcal{G}$ model structure associated to $\mathcal{T}$. In particular, if $i$ (resp. $u$ ) is a $\mathcal{T}$-equivalence, then $j$ (resp. $u^{\prime}$ ) is a $\mathcal{T}$-equivalence.

Proof. As the identity of $\operatorname{Comp}(\mathcal{A})$ is a left Quillen functor from the $\mathcal{G}$-model structure to the $\mathcal{G}$-model structure associated to $\mathcal{T}$, it is sufficient to prove that such a pushout square is a homotopy pushout square with respect to the $\mathcal{G}$-model structure. But the notion of a homotopy pushout square only depends on the weak equivalences, so that we are free to work with the injective model structure. The assertion is then trivial.

Proposition 4.6. The $\mathcal{T}$-equivalences are stable by filtering colimits in $\operatorname{Comp}(\mathcal{A})$.

Proof. We know that the quasi-isomorphisms are stable by filtering colimits (just because the filtering colimits are exact in any Grothendieck abelian category). This is equivalent to saying that for any filtering category $I$ and any functor $F$ from $I$ to $\operatorname{Comp}(\mathcal{A})$, the canonical map

$$
\underset{i}{\operatorname{holim}} F_{i} \longrightarrow \underset{i}{\longrightarrow} F_{i}
$$

is a quasi-isomorphism. But as the functor holim sends termwise $\mathcal{T}$-equivalences to $\mathcal{T}$-equivalences, this implies our assertion.

Proposition 4.7. A morphism of complexes of $\mathcal{A}$ is a fibration with respect to the $\mathcal{G}$-model structure associated to $\mathcal{T}$ if and only if it is a fibration with respect to the $\mathcal{G}$-model structure with $\mathcal{T}$-local kernel.

Proof. Let $p: K \longrightarrow L$ be a fibration with respect to the $\mathcal{G}$-model structure associated to $\mathcal{T}$. It has to be a fibration with respect to the $\mathcal{G}$-model structure and as the fibrations are stable by pullback, the map from the kernel of $p$ to 0 has to be a fibration with respect to the $\mathcal{G}$-model structure associated to $\mathcal{T}$. Hence the kernel of $p$ is $\mathcal{T}$-local. Conversely, let $p$ be a fibration with respect to the $\mathcal{G}$-model structure with $\mathcal{T}$-local kernel. We can factor $p=q i$ where $i: K \longrightarrow M$ is a $\mathcal{G}$-cofibration and a $\mathcal{T}$-equivalence and $q: M \longrightarrow L$ is a fibration with respect to the $\mathcal{G}$-model structure associated to $\mathcal{T}$. As the $\mathcal{G}$-model structure associated to $\mathcal{T}$ is right proper, the induced map from the kernel of $p$ to the kernel of $q$ is a $\mathcal{T}$-equivalence. But as these two kernels are $\mathcal{T}$-local, the map $\operatorname{ker} p \longrightarrow \operatorname{ker} q$ has to be a quasi-isomorphism, however both $p$ and $q$ are epimorphisms (this comes from the fact that they have the right lifting property with respect to the maps $0 \longrightarrow D^{n} E$ for $E$ in $\mathcal{G}$ and $n$ an integer). We thus 
have a morphism of distinguished triangles in $D(\mathcal{A})$

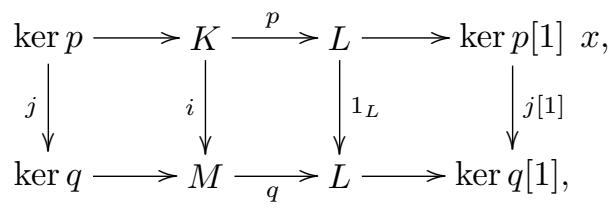

where $j$ is an quasi-isomorphism. This proves that $i$ is also a quasi-isomorphism. As it is also a $\mathcal{G}$-cofibration, it follows that $p$ is a retract of $q$. As $q$ is a fibration with respect to the $\mathcal{G}$-model structure associated to $\mathcal{T}, p$ must have the same property.

We recall here a folklore result that is quite useful to play the game between Bousfield localizations and Quillen functors; see [11].

Lemma 4.8. Let $G: \mathcal{C} \longrightarrow \mathcal{C}^{\prime}$ be a left Quillen functor between model categories. Suppose that we have a class $S$ of maps in $\mathcal{C}$ such that the left Bousfield localization of $\mathcal{C}$ with respect to $S$ exists. We denote by $\mathcal{C}_{S}$ the category $\mathcal{C}$ endowed with the model category structure obtained as the left Bousfield localization by S. Denote by $D$ a right adjoint to $G$. Then the following conditions are equivalent:

(a) The functor $G$ is a left Quillen functor from $\mathcal{C}_{S}$ to $\mathcal{C}^{\prime}$.

(b) For any fibrant object $X^{\prime}$ in $\mathcal{C}^{\prime}, D\left(X^{\prime}\right)$ is a fibrant object in $\mathcal{C}_{S}$.

(c) The functor $G$ sends the weak equivalences between cofibrant objects in $\mathcal{C}_{S}$ to weak equivalences.

(d) The functor $\mathbf{L} G: H o(\mathcal{C}) \longrightarrow H o\left(\mathcal{C}^{\prime}\right)$ sends the elements of $S$ to isomorphisms.

Proposition 4.9. Let $\mathcal{A}$ (resp. $\left.\mathcal{A}^{\prime}\right)$ be a Grothendieck abelian category with descent structure $(\mathcal{G}, \mathcal{H})$ (resp. $\left(\mathcal{G}^{\prime}, \mathcal{H}\right)$ ). Let $f^{*}: \mathcal{A}^{\prime} \rightarrow \mathcal{A}$ be a functor which has a right adjoint $f_{*}$ and satisfies descent. Consider a set $\mathcal{T}$ of $\mathcal{G}$-cofibrant complexes over $\mathcal{A}$, and a set $\mathcal{T}^{\prime}$ of $\mathcal{G}^{\prime}$-cofibrant complexes over $\mathcal{A}^{\prime}$ such that $f^{*}\left(\mathcal{T}^{\prime}\right) \subset \mathcal{T}$. Then the pair of adjoint functors

$$
f^{*}: \operatorname{Comp}\left(\mathcal{A}^{\prime}\right) \longrightarrow \operatorname{Comp}(\mathcal{A}) \quad \text { and } \quad f_{*}: \operatorname{Comp}(\mathcal{A}) \longrightarrow \operatorname{Comp}\left(\mathcal{A}^{\prime}\right)
$$

is a Quillen pair with respect to the $\mathcal{G}^{\prime}$-model structure associated to $\mathcal{T}^{\prime}$ and the $\mathcal{G}$ model structure associated to $\mathcal{T}$. In particular, we have a pair of adjoint functors

$$
\mathbf{L} f^{*}: D_{\mathcal{T}^{\prime}}\left(\mathcal{A}^{\prime}\right) \longrightarrow D_{\mathcal{T}}(\mathcal{A}) \quad \text { and } \quad \mathbf{R} f_{*}: D_{\mathcal{T}}(\mathcal{A}) \longrightarrow D_{\mathcal{T}^{\prime}}\left(\mathcal{A}^{\prime}\right)
$$

obtained as the left- and right derived functors of $f^{*}$ and $f_{*}$ respectively to the above model structures.

Proof. By virtue of Theorem 2.14, the functor $f^{*}$ is a left Quillen functor, so that it has a total left derived functor

$$
\mathbf{L} f^{*}: D\left(\mathcal{A}^{\prime}\right) \longrightarrow D_{\mathcal{T}}(\mathcal{A}) \text {. }
$$

Using Proposition 4.3 and condition (d) of Lemma 4.8, it is sufficient to check that $\mathbf{L} f^{*}$ sends the elements of $\mathcal{T}^{\prime}$ to null objects in $D_{\mathcal{T}}(\mathcal{A})$, which is obvious.

Remark 4.10. We have of course the same 2-functoriality results as in Remark 2.15 in the case where the obvious compatibilities are verified. 


\section{3.}

Let $\mathcal{A}$ be a closed symmetric monoidal Grothendieck category endowed with a weakly flat descent structure $(\mathcal{G}, \mathcal{H})$. A set $\mathcal{T}$ of $\mathcal{G}$-cofibrant complexes of $\mathcal{A}$ will be said to be flat (with respect to $\mathcal{G}$ ) if for any $E$ in $\mathcal{G}$ and any $T$ in $\mathcal{T}, E \otimes T$ is in $\mathcal{T}$.

Corollary 4.11. Let $\mathcal{A}$ be a closed symmetric monoidal Grothendieck category. We suppose that we have a weakly flat descent structure $(\mathcal{G}, \mathcal{H})$ on $\mathcal{A}$ and a flat set $\mathcal{T}$ of $\mathcal{G}$-cofibrant complexes of $\mathcal{A}$. Then the $\mathcal{G}$-model structure associated to $\mathcal{T}$ is a symmetric monoidal model category, and the localization functor $D(\mathcal{A}) \longrightarrow D_{\mathcal{T}}(\mathcal{A})$ is a symmetric monoidal functor.

If moreover $\mathcal{G}$ is flat, then this model category satisfies the monoid axiom of [20].

Proof. The proof of Lemma 3.6 can be modified slightly to prove that for any $\mathcal{G}$ cofibrant complex $A$ and any $T$ in $\mathcal{T}$, the map $0 \longrightarrow A \otimes T$ is a $\mathcal{T}$-equivalence. To prove the first assertion, we have to show that, given a $\mathcal{G}$-cofibration $A \longrightarrow B$ and a trivial $\mathcal{G}$-cofibration $C \longrightarrow D$ in the $\mathcal{G}$-model structure associated to $\mathcal{T}$, then the map

$A \otimes D \amalg_{A \otimes C} B \otimes C \longrightarrow B \otimes D$ is a $\mathcal{T}$-equivalence and a cofibration. It is sufficient to check this when $A \longrightarrow B$ is a generating $\mathcal{G}$-cofibration; see [12, Lemma 4.2.4]. In particular, we can assume that both $A$ and $B$ are $\mathcal{G}$-cofibrant. As we already know that the tensor product is well behaved with respect to cofibrations, we easily see that it is sufficient to prove that the tensor product of a trivial cofibration by a cofibrant object is a trivial cofibration, which follows from a new application of Lemma 4.8. Given any $\mathcal{G}$-cofibrant complex $A$, condition (d) of the lemma for $G=A \otimes(-)$ follows from the fact that, for any $T$ in $\mathcal{T}$, the map $0 \longrightarrow A \otimes T$ is a $\mathcal{T}$-equivalence.

If we assume that $\mathcal{G}$ is flat, then $(\mathcal{G}, \mathcal{H})$ is flat $(3.7)$. To prove the second assertion (the monoid axiom), we will consider the left Bousfield localization of the injective model structure on the category $\operatorname{Comp}(\mathcal{A})$ by the maps $0 \longrightarrow T[n]$ for any integer $n$ and any $T$ in $\mathcal{T}$. We will call the latter model category structure the $\mathcal{T}$-injective model structure. The identity of $\operatorname{Comp}(\mathcal{A})$ is a left Quillen equivalence from the $\mathcal{G}$-model structure associated to $\mathcal{T}$ to the $\mathcal{T}$-injective model structure. One easily deduces from this, from Propositions 4.5 and 4.6, and from the identification $D_{\mathcal{T}}(\mathcal{A})=D(\mathcal{A}) / \mathrm{T}(\mathcal{A})$ of Proposition 4.3 that the class of weak equivalences coincides for the two model structures associated to $\mathcal{T}$. In particular, the class of monomorphisms which are $\mathcal{T}$ equivalences is stable by transfinite composition and by pushout. It is then sufficient to prove that for any complex $C$, the functor $C \longmapsto C \otimes D$ is a left Quillen functor from the $\mathcal{G}$-model structure associated to $\mathcal{T}$ to the $\mathcal{T}$-injective model structure. From Proposition 3.4 and from Lemma 4.8 it follows that it is sufficient for this to prove that for any $\mathcal{T}$-equivalence $A \longrightarrow B$ between $\mathcal{G}$-cofibrant complexes, the map $C \otimes A \longrightarrow$ $C \otimes B$ is a $\mathcal{T}$-equivalence. But for any $\mathcal{G}$-cofibrant complex $K, C \otimes K$ is isomorphic to the derived tensor product of $C$ and $K$ (see Remark 3.8), so that by functoriality $C \otimes A \longrightarrow C \otimes B$ is an isomorphism in the homotopy category $D_{\mathcal{T}}(\mathcal{A})$. Hence the result.

Example 4.12. Consider the notation of Examples 2.4 and 2.13.

For a noetherian regular scheme $S$, we let $\mathcal{T}_{S}$ be the essentially small set of complexes of sheaves with transfers of the form $L_{S}\left(\mathbb{A}_{X}^{1}\right) \stackrel{p_{*}}{\longrightarrow} L_{S}(X)$ for any smooth $S$ scheme $X, p: \mathbb{A}_{X}^{1} \rightarrow X$ being the canonical projection. 
We define the category of motivic complexes over $S$ as the category

$$
D M^{e f f}(S):=D_{\mathcal{T}_{S}}\left(\mathcal{N}_{S}^{t r}\right)
$$

with the notation of Proposition 4.3.

A complex $C$ is $\mathcal{T}_{S}$-local (we will also say $\mathbb{A}^{1}$-local) if and only if its Nisnevich hypercohomology sheaves are homotopy invariant. Remark that if $C$ is Nisnevich fibrant, then it is $\mathbb{A}^{1}$-local if and only if, for any smooth $S$-scheme $X$, the map induced by the projection $C(X) \rightarrow C\left(\mathbb{A}_{X}^{1}\right)$ is a quasi-isomorphism, or equivalently, the cohomology presheaves of $C$ are homotopy invariant.

From Proposition 4.3, the category $D M^{\text {eff }}(S)$ is equivalent to the full subcategory of $D\left(\mathcal{N}_{S}^{t r}\right)$ made by the Nisnevich fibrant and $\mathbb{A}^{1}$-local complexes. This result has to be compared to the original definition by Voevodsky on motivic complexes over a perfect field (see [9, Chapter 5, following Proposition 3.1.13]. In fact, according to the strongest result of the theory (Theorem 3.1.12 of loc. cit.), over a perfect field, a complex of Nisnevich sheaves with transfers is $\mathbb{A}^{1}$-local if and only if its cohomology sheaves are homotopy invariant ${ }^{5}$.

According to Corollary 4.11 and Example 3.3, the category $D M^{e f f}(S)$ is symmetric monoidal. According to Proposition 4.9 and Example 2.16, for any morphism $f: T \rightarrow S$ of regular schemes, we have a pair of adjoint functors

$$
\mathbf{L} f^{*}: D M^{e f f}(S) \leftrightarrows D M^{e f f}(T): \mathbf{R} f_{*}
$$

and if $f$ is smooth, another pair

$$
\mathbf{L} f_{\sharp}: D M^{e f f}(T) \leftrightarrows D M^{e f f}(S): \mathbf{R} f^{*}=f^{*}=\mathbf{L} f^{*} .
$$

If we denote by $M_{S}(X)$ the object represented by a smooth scheme in $D M^{\text {eff }}(S)$, we have the fundamental relations

$$
\begin{aligned}
M_{S}(X) \otimes^{\mathbf{L}} M_{S}(Y) & =M_{S}\left(X \times_{S} Y\right), \\
\mathbf{R} f^{*} M_{S}(X) & =M_{T}\left(X \times_{S} T\right), \\
\mathbf{L} f_{\sharp} M_{T}(Y) & =M_{S}(Y)
\end{aligned}
$$

whenever it makes sense.

\section{Presentations of the derived category}

5.1 .

Let $R$ be a commutative ring with unit, and $\mathbb{A}$ an essentially small additive $R$-linear category. We denote by $\mathbb{A}$-Mod the category of right $\mathbb{A}$-modules, that is the category of $R$-linear additive functors from $\mathbb{A}^{o p}$ to the category of $R$-modules. The Yoneda embedding of $\mathbb{A}$ in $\mathbb{A}$-Mod is additive, $R$-linear and fully faithful. For any object $X$ of $\mathbb{A}$, we will also denote by $X$ the right $\mathbb{A}$-module represented by $X$.

Proposition 5.1. Let $\mathcal{G}_{\mathbb{A}}$ be a set of representative objects of $\mathbb{A}$. Then $\left(\mathcal{G}_{\mathbb{A}}, \varnothing\right)$ is a descent structure on the category of $\mathbb{A}$-modules. In other words, the $\mathcal{G}_{\mathbb{A}}$-model structure

\footnotetext{
${ }^{5}$ Among the notable facts this theorem implies is the description of the homotopy $t$-structure on
} $D M^{\text {eff }}(k)$ and the Gersten resolution for an object of its heart. 
on the category of complexes of $\mathbb{A}$-modules is the model category structure with the quasi-isomorphisms as weak equivalences and the epimorphisms as fibrations.

Proof. It is clear that $\left(\mathcal{G}_{\mathbb{A}}, \varnothing\right)$ is a descent structure on the category of $\mathbb{A}$-modules because $\mathcal{G}_{\mathbb{A}}$ is obviously a set of generators and because any complex of $\mathbb{A}$-modules is $\mathcal{G}_{\mathbb{A}}$-local. By definition of the $\mathcal{G}_{\mathbb{A}}$-model structure, the fibrations are the maps which have the right lifting property with respect to the maps $0 \longrightarrow D^{n} E$ for any $E$ in $\mathcal{G}_{\mathbb{A}}$ and any integer $n$. But as $D^{n} E$ represents the evaluation functor $K \longmapsto \operatorname{Hom}\left(E, K_{n}\right)$, this implies that the fibrations are exactly the epimorphisms of complexes of $\mathbb{A}$ modules.

\section{2 .}

Let $\mathcal{A}$ be a Grothendieck category and $(\mathcal{G}, \mathcal{H})$ a descent structure on $\mathcal{A}$. Let $\mathbb{A}$ be the full subcategory of $\mathcal{A}$ whose objects are the finite direct sums of elements of $\mathcal{G}$, and let $i$ be the inclusion functor. We have a functor

$$
i^{*}: \mathcal{A} \longrightarrow \mathbb{A}-\operatorname{Mod}
$$

defined by

$$
i^{*}(F)(X)=\operatorname{Hom}_{\mathcal{A}}(i(X), F)
$$

for any object $F$ of $\mathcal{A}$ and any object $X$ of $\mathbb{A}$. The functor $i^{*}$ has a left adjoint $i_{\text {! that }}$ can be defined as the left Kan extension of $i$ :

$$
i_{!}: \mathbb{A}-\operatorname{Mod} \longrightarrow \mathcal{A} \text {. }
$$

In particular, if $X$ is an object of $\mathbb{A}$, we have $i_{!}(X)=i(X)$ (recall that we denote also by $X$ the presheaf on $\mathbb{A}$ represented by $X$ ).

By virtue of the Gabriel-Popescu theorem $[10,15,17]$, we know that the functor $i^{*}$ is fully faithful and that the functor $i_{\text {! }}$ is exact (i.e. preserves finite limits).

Lemma 5.2. The functor $i_{!}: \operatorname{Comp}(\mathbb{A}-\operatorname{Mod}) \longrightarrow \operatorname{Comp}(\mathcal{A})$ is a left Quillen functor from the $\mathcal{G}_{\mathbb{A}}$-model structure (5.1) to the $\mathcal{G}$-model structure.

Proof. As $i_{\text {! }}$ preserves colimits, it is sufficient to prove that it sends the generating cofibrations (resp. trivial cofibrations) to cofibrations (resp. to trivial cofibrations). But for any object $X$ of $\mathbb{A}, E=i_{!}(X)=i(X)$ is in $\mathcal{G}$ and for any integer $n$ we have $i_{!}\left(D^{n} X\right)=D^{n} E$ and $i_{!}\left(S^{n} X\right)=S^{n} E$, so that the conclusion follows.

\section{3 .}

From now on we fix a regular cardinal $\alpha$ with the following properties:

(a) The functor $i^{*}$ preserves $\alpha$-filtered colimits.

(b) Any complex of $\mathbb{A}$-modules is a $\alpha$-filtered colimit of complexes of $\mathbb{A}$-modules which are of $\alpha$-small presentation ${ }^{6}$.

\footnotetext{
${ }^{6} \mathrm{An}$ object is of $\alpha$-small presentation if the functor $\operatorname{Hom}(X,-)$ preserves $\alpha$-filtered colimits
} 
We denote by $S$ a set of representatives of maps of shape

$$
\underset{j \in J}{\lim _{j \in}} i^{*} i_{!}\left(F_{j}\right) \longrightarrow i^{*} i_{!}\left(\underset{j \in J}{\lim } F_{j}\right),
$$

where $J$ is a $\alpha$-filtered small partially ordered set, and $F$ is a functor from $J$ to the category of $\mathbb{A}$-modules with values in objects of $\alpha$-small presentation, $J$ being itself of $\alpha$-small presentation in the category of partially ordered sets.

We will consider the $\mathcal{G}_{\mathbb{A}}$-model structure on $\operatorname{Comp}(\mathbb{A}$-Mod $)$ associated to the set of maps $\mathcal{T}=S \cup i^{*} \mathcal{H}$.

Lemma 5.3. For any complex $C$ of $\mathbb{A}$-modules, the map $C \longrightarrow i^{*} i_{!}(C)$ is a $\mathcal{T}$-equivalence.

Proof. As $\mathcal{T}$-equivalences are stable by filtered colimits (see 4.6), this follows formally from the definition of $S$ and from the fact that any filtered partially ordered set is a $\alpha$-filtered colimit of partially ordered sets of $\alpha$-small presentation.

Theorem 5.4. The functor $i_{!}: \operatorname{Comp}(\mathbb{A}-\operatorname{Mod}) \longrightarrow \operatorname{Comp}(\mathcal{A})$ is a Quillen equivalence from the $\mathcal{G}_{\mathbb{A}}$-model structure associated to $\mathcal{T}$ on $\operatorname{Comp}(\mathbb{A}$-Mod) to the $\mathcal{G}$-model structure on $\operatorname{Comp}(\mathcal{A})$.

Proof. Proposition 4.9 shows that we have defined a left Quillen functor. It remains to prove that the total left derived functor

$$
\mathbf{L} i_{!}: D_{\mathcal{T}}(\mathbb{A}-\operatorname{Mod}) \longrightarrow D(\mathcal{A})
$$

is an equivalence of categories. Note that, with the functor $i$ ! being exact and sending the elements of $\mathcal{T}$ to quasi-isomorphisms, it has to send $\mathcal{T}$-equivalences to quasiisomorphisms. The fully faithfulness of $i^{*}$ thus implies immediately the essential surjectivity of $\mathbf{L} i_{\text {! }}$. This also implies immediately the fact that $\mathbf{R} i^{*}$ is fully faithful. Using Lemma 5.3 and the fact that $i$ ! preserves monomorphisms and sends $\mathcal{T}$-equivalences to quasi-isomorphisms, one easily checks that the class of maps of $\operatorname{Comp}(\mathcal{A})$, which are monomorphisms and whose image by $i^{*}$ is a $\mathcal{T}$-equivalence, is stable by pushouts, by transfinite compositions, and by retracts. Using again Lemma 5.3 and the fact that $i^{*} \mathcal{H}$ belongs to $\mathcal{T}$ (by definition), this implies, using the small object argument, that $i^{*}$ sends any trivial cofibration of $\operatorname{Comp}(\mathcal{A})$ to a $\mathcal{T}$-equivalence. By Ken Brown's lemma, this implies that $i^{*}$ preserves weak equivalences between $\mathcal{G}$-cofibrant complexes. If $C$ is a $\mathcal{G}_{\mathbb{A}}$-cofibrant complex of $\mathbb{A}$-modules, then we thus have a canonical isomorphism $i^{*} i_{!}(C) \longrightarrow \mathbf{R} i^{*}\left(i_{!}(C)\right)$ in $D(\mathcal{A})$, so that the unit map $C \longrightarrow \mathbf{R} i^{*}\left(i_{!}(C)\right)$ has to be an isomorphism by Lemma 5.3 .

\section{4 .}

Suppose that a morphism $u: K \longrightarrow L$ is a $\mathcal{G}$-surjection if for any $E$ in $\mathcal{G}$, the map

$$
\operatorname{Hom}_{\mathcal{A}}(E, K) \longrightarrow \operatorname{Hom}_{\mathcal{A}}(E, L)
$$

is surjective.

Corollary 5.5. A morphism of complexes of $\mathcal{A}$ is a fibration for the $\mathcal{G}$-model structure if and only if it is a degreewise $\mathcal{G}$-surjection with $\mathcal{G}$-local kernel. 
Proof. Any fibration for the $\mathcal{G}$-model structure on $\operatorname{Comp}(\mathcal{A})$ is a $\mathcal{G}$-surjection with $\mathcal{G}$-local kernel. Conversely, it follows from Theorem 2.5 and Propositions 4.7 and 5.1 that a morphism $p: X \longrightarrow Y$ of complexes of $\mathcal{A}$ is degreewise $\mathcal{G}$-surjective with $\mathcal{G}$ local kernel if and only if $i^{*}(p)$ is a fibration with respect to the $\mathcal{G}_{\mathbb{A}}$-model structure associated to $\mathcal{T}$. This implies that $p$ has the right lifting property with respect to trivial cofibrations between cofibrant objects. But, from Lemma 2.7, it follows that all the generating trivial cofibrations are cofibrations between cofibrant objects. Hence the result.

Corollary 5.6. Let $\mathcal{A}$ be a Grothendieck category endowed with a descent structure $(\mathcal{G}, \mathcal{H})$ and a set $\mathcal{T}$ of complexes of $\mathcal{A}$. A morphism of complexes of $\mathcal{A}$ is a fibration with respect to the $\mathcal{G}$-model structure associated to $\mathcal{T}$ if and only if it is degreewise $\mathcal{G}$-surjective with a $\mathcal{G}$-local and $\mathcal{T}$-local kernel.

Proof. This follows from Proposition 4.7 and Corollary 5.5.

Remark 5.7. The preceding result implies that we can describe a generating set of trivial cofibrations of the $\mathcal{G}$-model structure associated to $\mathcal{T}$ on $\operatorname{Comp}(\mathcal{A})$ : just add to the generating trivial cofibrations of the $\mathcal{G}$-model structure on $\operatorname{Comp}(\mathcal{A})$ the maps $T^{\prime} \oplus T^{\prime}[n] \longrightarrow \operatorname{Cyl}\left(T^{\prime}\right)[n]$ where $n$ runs over the integers, $T$ over the set $\mathcal{T}$, and where $T^{\prime}$ is a choice of a $\mathcal{G}$-cofibrant resolution of $T$.

\section{How to get compact generators}

\section{1 .}

Recall that, for a triangulated category $\mathcal{T}$, an object $X$ is compact if for any set $I$ and any family of objects $Y_{i}, i \in I$, in $\mathcal{T}$, the map

$$
\bigoplus_{i \in I} \operatorname{Hom}_{\mathcal{T}}\left(X, Y_{i}\right) \longrightarrow \operatorname{Hom}_{\mathcal{T}}\left(X, \bigoplus_{i \in I} Y_{i}\right)
$$

is surjective (hence bijective). One checks easily that the full subcategory of compact objects in $\mathcal{T}$ is a triangulated subcategory of $\mathcal{T}$.

Consider a Grothendieck abelian category $\mathcal{A}$. We say that a descent structure $(\mathcal{G}, \mathcal{H})$ on $\mathcal{A}$ is bounded if the complexes $H$ in $\mathcal{H}$ are all bounded and degreewise finite sums of objects in $\mathcal{G}$. The following lemma is straightforward.

Lemma 6.1. Let $R$ be a commutative ring with unit and $\mathbb{A}$ be an $R$-linear additive category. Any bounded complex of $\mathbb{A}$-modules which is degreewise a finite sum of representable $\mathbb{A}$-modules is compact in the triangulated category $K(\mathbb{A}$-Mod).

\section{2 .}

Consider a bounded descent structure $(\mathcal{G}, \mathcal{H})$ on $\mathcal{A}$. Let $\mathbb{A}$ be the full subcategory of $\mathcal{A}$ that consists of finite sums of objects in $\mathcal{G}$. We define the triangulated category $D_{c}(\mathcal{A})$ as follows: Consider the bounded homotopy category of $\mathbb{A}$, denoted by $K^{b}(\mathbb{A})$. The previous lemma implies easily that the triangulated category of compact objects in $K\left(\mathbb{A}\right.$-Mod) contains the pseudo-abelianization ${ }^{7}$ of $K^{b}(\mathbb{A})$. We can consider the

\footnotetext{
${ }^{7}$ This means the idempotent completion: we add formally the kernel and cokernel of any projector.
} 
objects in $\mathcal{H}$ as bounded complexes in $\mathbb{A}$. Let $\langle\mathcal{H}\rangle$ be the thick subcategory of $K^{b}(\mathbb{A})$ generated by the objects in $\mathcal{H}$, and define $D_{c}(\mathcal{A})$ to be the pseudo-abelianization of the Verdier quotient of $K^{b}(\mathbb{A})$ by $\langle\mathcal{H}\rangle$. From a general result of Balmer and Schlichting [2] it follows that $D_{c}(\mathcal{A})$ is canonically endowed with a structure of triangulated category such that the functor $K^{b}(\mathbb{A}) \longrightarrow D_{c}(\mathcal{A})$ is triangulated.

Theorem 6.2. Assume, for any object $X$ in $\mathcal{G}$, that the functor $\operatorname{Hom}_{\mathcal{A}}(X,-)$ preserves small direct sums. Any object in $\mathcal{G}$, seen as a complex concentrated in degree 0 , is a compact object in $D(\mathcal{A})$. Moreover, the functor

$$
\mathbf{L} i_{!}: D(\mathbb{A}-\mathrm{Mod}) \longrightarrow D(\mathcal{A})
$$

induces an equivalence of triangulated categories between the category of compact objects of $D(\mathcal{A})$ and the category $D_{c}(\mathcal{A})$.

Proof. Under the hypothesis made on the elements of $\mathcal{G}$, the functor $i_{\text {! }}$ is fully faithful on the full subcategory of $\operatorname{Comp}\left(\mathbb{A}\right.$-Mod) made of the $\mathcal{G}_{\mathbb{A}}$-cofibrant complexes. This comes from the facts that $i$ is fully faithful by definition and that if $C$ is a $\mathcal{G}_{\mathbb{A}}$-cofibrant complex of $\mathbb{A}$-modules, then for any integer $n, C^{n}$ is a direct summand of a direct sum of representable presheaves on $\mathbb{A}$. Any bounded complex which is degreewise a direct sum of representable $\mathbb{A}$-modules is in the essential image of $i_{\text {! }}$. In particular, $\mathcal{H}$ is in the essential image of $i_{\text {! }}$.

Let $C$ be a $\mathcal{G}_{\mathbb{A}}$-cofibrant $\mathbb{A}$-module. Considering the $\mathcal{G}_{\mathbb{A}}$-model structure associated to $i^{*}(\mathcal{H})$ on $\operatorname{Comp}\left(\mathbb{A}\right.$-Mod), one can choose a $i^{*}(\mathcal{H})$-flasque resolution of $C$, i.e. a quasiisomorphism $C \longrightarrow C^{\prime}$, with $C^{\prime} \mathcal{G}_{\mathbb{A}}$-cofibrant and $i^{*}(\mathcal{H})$-flasque. Using the exactness of $i_{\text {! }}$ and its fully faithfulness on $\mathcal{G}_{\mathbb{A}}$-cofibrant complexes, we see easily that the map $i_{!}(C) \longrightarrow i_{!}\left(C^{\prime}\right)$ is a quasi-isomorphism whose target is $\mathcal{H}$-flasque. In particular, for any object $X$ in $\mathcal{G}$ and any integer $n$, the map

$$
\operatorname{Hom}_{D_{i^{*}(\mathcal{H})}(\operatorname{Comp}(\mathbb{A}-\operatorname{Mod}))}(X, C[i]) \longrightarrow \operatorname{Hom}_{D(\mathcal{A})}\left(i_{!}(X), i_{!}(C)[i]\right)
$$

is an isomorphism. As $\mathcal{G}$ form a set of generators of the derived category of $\mathcal{A}$, we deduce from this that the functor

$$
\mathbf{L} i_{!}=i_{!}: D_{i^{*}(\mathcal{H})}(\operatorname{Comp}(\mathbb{A}-\mathrm{Mod})) \longrightarrow D(\mathcal{A})
$$

is fully faithful. As we already know it is essentially surjective (this follows immediately from Theorem 5.4, or can be checked directly: $\mathcal{G}$ is in the essential image of $i_{\text {! }}$ by definition), we can end the proof by using Lemma 6.1 and Thomason's theorem; see [19, Theorem 4.4.9].

Example 6.3. Consider the notation of Example 4.12.

For any regular noetherian scheme $S$ of finite Krull dimension, there exists a bounded descent structure $\left(\mathcal{G}_{S}, \mathcal{H}_{S}^{\prime}\right)$ on the category of Nisnevich sheaves with transfers: using the Nisnevich version of the Brown-Gersten theorem [18, Lemma 1.17, p. 101], we can take $\mathcal{H}_{S}^{\prime}$ to be the set of complexes of shape

$$
\cdots \longrightarrow 0 \longrightarrow L_{S}\left(U \times_{X} V\right) \longrightarrow L_{S}(U) \oplus L_{S}(V) \longrightarrow L_{S}(X) \longrightarrow 0 \longrightarrow \cdots
$$

associated to distinguished squares in the sense of [18, Definition 1.3, p. 96]. The $\mathcal{H}_{S}^{\prime}$-flasque complexes are then exactly those which satisfy the Nisnevich version of the Mayer-Vietoris long exact sequence. 
Recall that the category of finite correspondences $\mathcal{S} m_{S}^{\text {cor }}$ over $S$ from [8, 4.1.19] is the category whose objects are smooth $S$-schemes and morphisms from $X$ to $Y$ are cycles in $X \times{ }_{S} Y$ which support is finite equidimensional over $X$. It is additive and symmetric monoidal.

According to 6.2 , we consider the thick subcategory $\left\langle\mathcal{H}_{S}^{\prime}, \mathcal{T}_{S}\right\rangle$ of $K^{b}\left(\mathcal{S} m_{S}^{\text {cor }}\right)$ generated by the classes $\mathcal{H}_{S}^{\prime}$ and $\mathcal{T}_{S}$. We denote by $D M_{g m}^{\text {eff }}(S)$ the pseudo-abelianization of $K^{b}\left(\mathcal{S} m_{S}^{c o r}\right) /\left\langle\mathcal{H}_{S}^{\prime}, \mathcal{T}_{S}\right\rangle$.

The preceding theorem implies we have a canonical fully faithful functor

$$
D M_{g m}^{e f f}(S) \rightarrow D M^{e f f}(S)
$$

whose essential image is the category of compact objects of $D M^{e f f}(S)$.

\section{Stabilization and symmetric spectra}

\section{1.}

Let $\mathcal{A}$ be a Grothendieck abelian category. We suppose that $\mathcal{A}$ is endowed with a symmetric monoidal structure. We denote by $\otimes$ and $\mathbf{1}$ the tensor product and the unit respectively. We also suppose that for any object $X$ of $\mathcal{A}$, the functor

$$
Y \longmapsto X \otimes Y
$$

preserves colimits.

\section{2 .}

We will consider in this subsection $G$-objects of $\mathcal{A}$ for various (finite) groups $G$, that are object $A$ of $\mathcal{A}$ equipped with a representation $G \longrightarrow \operatorname{Aut}_{\mathcal{A}}(A)$.

We introduce the following notation:

If $A$ is an object of $\mathcal{A}$, we put $G \times A=\bigoplus_{g \in G} A$ considered as a $G$-object via the permutation isomorphisms of the summands.

If $H$ is a subgroup of $G$, and $A$ is an $H$-object, then $G \times A$ has two actions of $H$ : the first one, say $\gamma$, is obtained via the inclusion $H \subset G$, and the second one denoted by $\gamma^{\prime}$, is obtained using the structural action of $H$ on $A$, making $H$ acting diagonally on $G \times A$. We define $G \times{ }_{H} A$ as the equalizer of the family of morphisms $\left(\gamma_{\sigma}-\gamma_{\sigma}^{\prime}\right)_{\sigma \in H}$, and consider it equipped with its induced action of $G$.

We write $\mathfrak{S}_{n}$ for the symmetric group (that is the group of automorphisms of the set with $n$ elements, $n \geqslant 0$ ), and we make the following definition.

Definition 7.1. A symmetric sequence of $\mathcal{A}$ is a sequence $\left(A_{n}\right)_{n \in \mathbb{N}}$ such that for each $n \in \mathbb{N}, A_{n}$ is a $\mathfrak{S}_{n}$-object of $\mathcal{A}$. A morphisms of symmetric sequences is a degreewise equivariant morphism in $\mathcal{A}$.

\section{3 .}

For an integer $n \geqslant 0$, we denote by $\underline{n}$ the set of integers $i$ such that $1 \leqslant i \leqslant n$. We then introduce the category $\mathfrak{S}$ as follows. The objects are the sets $\underline{n}$ for $n \geqslant 0$, and the morphisms are the bijections between such sets. Then, $\mathfrak{S}$ is a groupoid and the category of symmetric sequences can be described as the category of functors from $\mathfrak{S}$ to $\mathcal{A}$. We use the notation $\mathcal{A}^{\mathfrak{S}}$ to denote the category of symmetric sequences of $\mathcal{A}$. 
We have a full embedding

$$
\mathcal{A} \longrightarrow \mathcal{A}^{\mathfrak{S}}, \quad X \longmapsto X\{0\}
$$

which is a left adjoint to the 0 -evaluation functor $\mathcal{A}^{\mathfrak{S}} \longrightarrow \mathcal{A}, A_{*} \longmapsto A_{0}$. More precisely, if $X$ is an object of $\mathcal{A}$, then $X\{0\}$ is the symmetric sequence defined by $X\{0\}_{0}=X$ and $X\{0\}_{n}=0$ for $n>0$.

Consider an integer $i \in \mathbb{N}$ and a symmetric sequence $A_{*}$ of $\mathcal{A}$. We put

$$
A_{*}\{-i\}=n \longmapsto \begin{cases}\mathfrak{S}_{n} \times \mathfrak{S}_{n-i} A_{n-i} & \text { if } n \geqslant i \\ 0 & \text { otherwise. }\end{cases}
$$

This defines an endofunctor on $\mathcal{A}^{\mathfrak{S}}$, and we have

$$
A_{*}\{-i\}\{-j\} \simeq A_{*}\{-i-j\} .
$$

For an object $X$ of $\mathcal{A}$, we will write $X\{-i\}=X\{0\}\{-i\}$. For $i \geqslant 0$, the functor $A_{*} \longmapsto A_{*}\{-i\}$ has a right adjoint $A_{*} \longmapsto A_{*}\{i\}$ which is defined by $A_{*}\{i\}_{n}=A_{n+i}$, where the action of $\mathfrak{S}_{n}$ on $A_{n+i}$ is induced by the canonical inclusion of $\mathfrak{S}_{n}$ in $\mathfrak{S}_{n+i}$.

Remark that for any integer $n \in \mathbb{N}$, the functor $\mathcal{A} \longrightarrow \mathcal{A}^{\mathfrak{S}}, X \longmapsto X\{-n\}$ is left adjoint to the $n$-evaluation functor $\mathcal{A}^{\mathfrak{S}} \longrightarrow \mathcal{A}, A_{*} \longmapsto A_{n}$. Moreover, the collection of functors $\mathcal{A}^{\mathfrak{S}} \stackrel{E v_{n}}{\longrightarrow} \mathcal{A}$ preserves every limits and colimits and is conservative.

We now define a symmetric monoidal structure on $\mathcal{A}^{\mathfrak{S}}$.

First of all, let us introduce a variant of $\mathfrak{S}$ by considering the category $\mathfrak{S}^{\prime}$ of finite sets with morphisms only the bijections. The canonical functor $\mathfrak{S} \longrightarrow \mathfrak{S}^{\prime}$ is thus an equivalence of categories. This means that the restriction functor $\mathcal{A}^{\mathfrak{S}^{\prime}} \longrightarrow \mathcal{A}^{\mathfrak{S}}$ is an equivalence of categories.

Let now $E, F: \mathfrak{S}^{\prime} \longrightarrow \mathcal{A}$ be functors. We define a functor

$$
\begin{aligned}
E \otimes{ }^{\mathfrak{S}} F: \mathfrak{S}^{\prime} & \longrightarrow \mathcal{A} \\
N & \longmapsto \bigoplus_{N=P \sqcup Q} E(P) \otimes F(Q) .
\end{aligned}
$$

This makes $\mathcal{A}^{\mathfrak{S}^{\prime}}$, and thus $\mathcal{A}^{\mathfrak{S}}$ into a symmetric monoidal category, the unit being the symmetric sequence $\mathbf{1}\{0\}$, and the symmetry isomorphism being induced by the symmetry isomorphism of $\otimes$ in $\mathcal{A}$. It is then straightforward to check that the canonical functor $X \longmapsto X\{0\}$ is a symmetric monoidal functor from $\mathcal{A}$ to $\mathcal{A}^{\mathfrak{S}}$.

Remark 7.2. The above definition is only a way to simplify the combinatorics of $\otimes^{\mathfrak{G}}$. To help the reader using it, we point out that to give a morphism of symmetric objects $A_{*} \otimes^{\mathfrak{S}} B_{*} \longrightarrow C_{*}$ is equivalent to giving families of maps $A_{p} \otimes B_{q} \longrightarrow C_{p+q}$, which are $\mathfrak{S}_{p} \times \mathfrak{S}_{q}$-equivariant, the action on the right-hand side being given by the canonical inclusion $\mathfrak{S}_{p} \times \mathfrak{S}_{q} \longrightarrow \mathfrak{S}_{p+q}$.

\section{4 .}

Let $S$ be an object of $\mathcal{A}$. We define $S^{\otimes n}$ as

$$
S^{\otimes n}=\underbrace{S \otimes \cdots \otimes S}_{n \text { times }} .
$$


The group of permutations $\mathfrak{S}_{n}$ acts on $S^{\otimes n}$ by the structural symmetry isomorphisms of the monoidal structure. We denote by $\operatorname{Sym}(S)$ the symmetric sequence $\left(S^{\otimes n}\right)_{n \in \mathbb{N}}$ obtained by considering the above actions.

Moreover, the canonical isomorphism functor

$$
S^{\otimes p} \otimes S^{\otimes q} \longrightarrow S^{\otimes p+q}
$$

obtained using the associativity isomorphism, is $\mathfrak{S}_{p} \times \mathfrak{S}_{q}$-equivariant. Thus, this defines a morphism

$$
\operatorname{Sym}(S) \otimes{ }^{\mathfrak{S}} \operatorname{Sym}(S) \rightarrow \operatorname{Sym}(S) .
$$

One checks now that with this structural morphism, $\operatorname{Sym}(S)$ is a commutative monoid in the category $\mathcal{A}^{\mathfrak{S}}$ (the unit is defined by the identity of $\mathbf{1}$ ). Recall that the symmetric sequence $S\{0\}$ defined by $S\{0\}_{0}=S$ and $S\{0\}_{n}=0$ for $n>0$, and remember that $S\{-1\}=S\{0\}\{-1\}$. Thus $S\{-1\}$ is the symmetric sequence defined by $S\{-1\}_{n}=0$ if $n \neq 1$ and $S\{-1\}_{1}=S$. One has a natural morphism of symmetric sequences

$$
S\{-1\} \longrightarrow \operatorname{Sym}(S)
$$

induced by the identity of $S$. One can check that $\operatorname{Sym}(S)$ is the free commutative monoid generated by $S\{-1\}$ in $\mathcal{A}^{\mathfrak{S}}$. In other words, for any commutative monoid object $M$ in the category of symmetric sequences, any morphism from $S$ to $M_{1}$ in $\mathcal{A}$ can be extended uniquely into a morphism of commutative monoids from $\operatorname{Sym}(S)$ to $M$ in $\mathcal{A}^{\mathfrak{S}}$ (exercise left to the reader).

Given an object $S$ of $\mathcal{A}$, we define the category of symmetric $S$-spectra as the category of modules in $\mathcal{A}^{\mathfrak{S}}$ over the commutative monoid $\operatorname{Sym}(S)$. In explicit terms, a symmetric $S$-spectra $E$ is a collection $\left(E_{n}, \sigma_{n}\right)_{n \geqslant 0}$, where, for any $n \geqslant 0, E_{n}$ is an object of $\mathcal{A}$ and $\sigma_{n}: S \otimes E_{n} \longrightarrow E_{n+1}$ is a morphism in $\mathcal{A}$ such that the induced map obtained by composition

$$
S^{\otimes m} \otimes E_{n} \longrightarrow S^{\otimes m-1} \otimes E_{n+1} \longrightarrow \cdots \longrightarrow S \otimes E_{m+n-1} \longrightarrow E_{m+n}
$$

is $\mathfrak{S}_{m} \times \mathfrak{S}_{n}$-equivariant for any integers $n \geqslant 0, m \geqslant 0$. A morphism of symmetric $S$ spectra $u:\left(E_{n}, \sigma_{n}\right) \longrightarrow\left(F_{n}, \tau_{n}\right)$ is a collection of $\mathfrak{S}_{n}$-equivariant maps $u_{n}: E_{n} \longrightarrow F_{n}$ such that the square

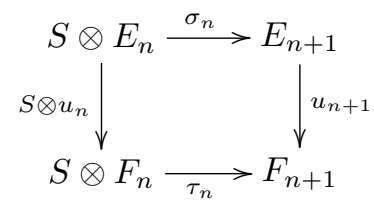

commutes in $\mathcal{A}$ for any integer $n \geqslant 0$.

\section{5 .}

We consider now a closed symmetric monoidal Grothendieck abelian category $\mathcal{A}$ endowed with a weakly flat descent structure $(\mathcal{G}, \mathcal{H})$ and a flat set $\mathcal{T}$ of complexes of $\mathcal{A}$ (see 4.3). By virtue of Corollary 4.11, the category $\operatorname{Comp}(\mathcal{A})$ is endowed with a closed model category structure associated to $\mathcal{T}$ which is compatible with the tensor product. Let $S$ be a $\mathcal{G}$-cofibrant complex. We want $S$ to be invertible in the following sense: we want the derived tensor product by $S$ to be an equivalence of categories. 
For this purpose, we will embed the category $\operatorname{Comp}(\mathcal{A})$ in the category of symmetric $S$-spectra by a symmetric monoidal functor, and we will define a model category structure on the category of symmetric $S$-spectra for which the tensor product by $S$ will be a left Quillen equivalence. We will first have to study the homotopy theory of complexes of symmetric sequences associated to $\mathcal{T}$.

The category $\operatorname{Comp}(\mathcal{A})^{\mathfrak{S}}$ of symmetric sequences in $\operatorname{Comp}(\mathcal{A})$ is canonically equivalent to the category $\operatorname{Comp}\left(\mathcal{A}^{\mathfrak{S}}\right)$ of complexes of $\mathcal{A}^{\mathfrak{S}}$. Define $\mathcal{G}^{\mathfrak{S}}$ to be the class of objects $E\{-i\}$ for $E$ in $\mathcal{G}$ and $i \geqslant 0$, and $\mathcal{H}^{\mathfrak{S}}$ (resp. $\mathcal{T}^{\mathfrak{S}}$ ) the class of complexes $H\{-i\}$ for $H$ in $\mathcal{H}$ (resp. $T\{-i\}$ for $T$ in $\mathcal{T}$ ) and any integer $i \geqslant 0$.

Lemma 7.3. $\mathcal{G}^{\mathfrak{S}}$ is a generating family of $\mathcal{A}^{\mathfrak{S}}$. If moreover $\mathcal{G}$ is flat, then $\mathcal{G}^{\mathfrak{S}}$ is flat.

Proof. We first have to show that $\mathcal{G}^{\mathfrak{S}}$ is a generating family for $\mathcal{A}^{\mathfrak{S}}$. But for a symmetric sequence $A_{*}$, we have

$$
\operatorname{Hom}_{\mathcal{A} \mathfrak{S}}\left(E\{-i\}, A_{*}\right)=\operatorname{Hom}_{\mathcal{A}}\left(E, A_{i}\right) .
$$

As the family of evaluation functors $\mathcal{A}^{\mathfrak{S}} \stackrel{E v_{n}}{\longrightarrow} \mathcal{A}$ is conservative and as $\mathcal{G}$ is a generating family of $\mathcal{A}$, this implies that $\mathcal{G}^{\mathfrak{S}}$ is a generating family of $\mathcal{A}^{\mathfrak{S}}$. It is easy to see that $\mathcal{G}^{\mathfrak{S}}$ is flat: this comes from the fact that for any objects $X$ and $Y$ of $\mathcal{A}$ and any integers $i, j \geqslant 0$, we have $X\{-i\} \otimes^{\mathfrak{S}} Y\{-j\}=(X \otimes Y)\{-i-j\}$.

Lemma 7.4. If $C$ is a $\mathcal{G}$-cofibrant complex (resp. an acyclic complex), then $C\{-i\}$ is $\mathcal{G}^{\mathfrak{S}}$-cofibrant (resp. acyclic).

Proof. This follows from the fact that the functors $\mathcal{A} \longrightarrow \mathcal{A}^{\mathfrak{S}}, X \longmapsto X\{-i\}$ preserve colimits and are exact.

Lemma 7.5. For any complex $C$ of $\mathcal{A}$ and any complex $K_{*}$ of $\mathcal{A}^{\mathfrak{S}}$, we have canonical isomorphisms for $i \geqslant 0$

$$
\operatorname{Hom}_{K\left(\mathcal{A}^{\mathfrak{S}}\right)}\left(C\{-i\}, K_{*}\right) \simeq \operatorname{Hom}_{K(\mathcal{A})}\left(C, K_{i}\right) .
$$

Proof. We have $\operatorname{Hom}_{\operatorname{Comp}\left(\mathcal{A}^{\mathfrak{S}}\right)}\left(C\{-i\}, K_{*}\right) \simeq \operatorname{Hom}_{\mathrm{Comp}(\mathcal{A})}\left(C, K_{i}\right)$, and this isomorphism is compatible with the cochain homotopy relation.

Lemma 7.6. For any complex $C$ of $\mathcal{A}$ and any complex $K_{*}$ of $\mathcal{A}^{\mathfrak{S}}$, we have canonical isomorphisms for $i \geqslant 0$

$$
\operatorname{Hom}_{D\left(\mathcal{A}^{\mathfrak{S}}\right)}\left(C\{-i\}, K_{*}\right) \simeq \operatorname{Hom}_{D(\mathcal{A})}\left(C, K_{i}\right) .
$$

Proof. This follows from these two facts: The functor $\operatorname{Comp}(\mathcal{A}) \longrightarrow \operatorname{Comp}\left(\mathcal{A}^{\mathfrak{S}}\right)$, $C \longmapsto C\{-i\}$ is a left Quillen functor for the injective model structures on $\operatorname{Comp}(\mathcal{A})$ and $\operatorname{Comp}\left(\mathcal{A}^{\mathfrak{S}}\right)$ and the functor $\operatorname{Comp}\left(\mathcal{A}^{\mathfrak{S}}\right) \longrightarrow \operatorname{Comp}(\mathcal{A}), C_{*} \longmapsto C_{i}$ preserves quasi-isomorphisms. Indeed, this shows that it is sufficient to prove the result for $K_{*}$ fibrant with respect to the injective model structure. But then this reduces to Lemma 7.5.

Proposition 7.7. $\left(\mathcal{G}^{\mathfrak{S}}, \mathcal{H}^{\mathfrak{S}}\right)$ is a weakly flat descent structure on $\mathcal{A}^{\mathfrak{S}}$ and the family $\mathcal{T}^{\mathfrak{S}}$ of the $T\{-i\}$ 's for $T$ in $\mathcal{T}$ and $i \geqslant 0$ is flat. Moreover, a complex of symmetric sequences $K_{*}$ is $\mathcal{G}^{\mathfrak{S}}$-local (resp. $\mathcal{H}^{\mathfrak{S}}$-flasque, resp. $\mathcal{T}^{\mathfrak{S}}$-local) if and only if for any integer $i \geqslant 0, K_{i}$ is $\mathcal{G}$-local (resp. $\mathcal{H}$-flasque, resp. $\mathcal{T}$-local).

If $\mathcal{G}$ is flat, then the descent structure $\left(\mathcal{G}^{\mathfrak{S}}, \mathcal{H}^{\mathfrak{S}}\right)$ is flat. 
Proof. It follows from Lemmata 7.5 and 7.6 that a complex of symmetric sequences $K_{*}$ is $\mathcal{G}^{\mathfrak{S}}$-local (resp. $\mathcal{H}^{\mathfrak{S}}$-flasque, resp. $\mathcal{T}^{\mathfrak{S}}$-local) if and only if for any integer $i \geqslant 0$, $K_{i}$ is $\mathcal{G}$-local (resp. $\mathcal{H}$-flasque, resp. $\mathcal{T}$-local). This implies that $\left(\mathcal{G}^{\mathfrak{S}}, \mathcal{H}^{\mathfrak{S}}\right)$ is a descent structure. We deduce easily from Lemma 7.4 that it is weakly flat. If moreover $\mathcal{G}$ is flat, then $\left(\mathcal{G}^{\mathfrak{S}}, \mathcal{H}^{\mathfrak{S}}\right)$ is a flat descent structure: this comes from Lemmata 7.3 and 7.4 and from Proposition 3.7.

Proposition 7.8. A morphism of complexes of symmetric sequences $u_{*}: K_{*} \longrightarrow L_{*}$ is a $\mathcal{T}^{\mathfrak{S}}$-equivalence (resp. a fibration with respect to the $\mathcal{G}^{\mathfrak{S}}$-model structure associated to $\mathcal{T}^{\mathfrak{S}}$ ) if and only if for any integer $i \geqslant 0$, the map $u_{i}: K_{i} \longrightarrow L_{i}$ is a $\mathcal{T}$ equivalence (resp. a fibration with respect to the $\mathcal{G}$-model structure associated to $\mathcal{T}$ ).

Proof. The characterization of fibrations follows from Proposition 7.7 and Corollary 5.6. If $i \geqslant 0$ is an integer, then the evaluation functor at $i$,

$$
\operatorname{Comp}(\mathcal{A})^{\mathfrak{S}} \longrightarrow \operatorname{Comp}(\mathcal{A}), \quad A_{*} \longmapsto A_{i}
$$

sends the $\mathcal{G}^{\mathfrak{S}}$-cofibrations (resp. the trivial $\mathcal{G}^{\mathfrak{S}}$-cofibrations) to $\mathcal{G}$-cofibrations (resp. trivial $\mathcal{G}$-cofibrations). The class of $\mathcal{G}^{\mathfrak{S}}$-cofibrations (resp. of trivial $\mathcal{G}^{\mathfrak{S}}$-cofibrations) is generated by maps of the form $u\{-j\}: A\{-j\} \longrightarrow B\{-j\}$, where $j \geqslant 0$ is an integer and $u$ is a $\mathcal{G}$-cofibration (resp. a trivial $\mathcal{G}$-cofibration); see and apply 2.3 and Remark 5.7 to $\mathcal{A}^{\mathfrak{S}}$. As the evaluation functor at $i$ preserves colimits, it is sufficient to check that for any $\mathcal{G}$-cofibration (resp. trivial $\mathcal{G}$-cofibration) $u: A \longrightarrow B$ and any integer $j \leqslant 0$, the morphism $u\{-j\}_{i}: A\{-j\}_{i} \longrightarrow B\{-j\}_{i}$ is a $\mathcal{G}$-cofibration (resp. a trivial $\mathcal{G}$-cofibration). But for $i \neq j$, everything is zero, so that we only have to deal with the case $i=j$. Then the map $u\{-i\}_{i}$ is

$$
\bigoplus_{\mathfrak{S}_{i}} A=\mathfrak{S}_{i} \times A \longrightarrow \mathfrak{S}_{i} \times B=\bigoplus_{\mathfrak{S}_{i}} B x
$$

Our claim thus follows from the fact that $\mathcal{G}$-cofibrations (resp. trivial $\mathcal{G}$-cofibrations) are stable by (finite) direct sums. Let $u_{*}: K_{*} \longrightarrow L_{*}$ be a morphism of symmetric sequences. Using the existence of factorizations into a trivial cofibration followed by a fibration in the $\mathcal{G}^{\mathfrak{S}}$-model structure associated to $\mathcal{T}^{\mathfrak{S}}$, one can produce a diagram

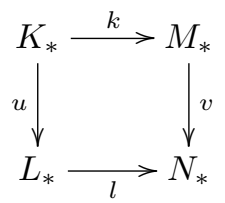

in which $k$ and $l$ are trivial cofibrations and $M_{*}$ and $N_{*}$ are fibrant. As $k$ and $l$ are both termwise $\mathcal{T}$-equivalences and $\mathcal{T}^{\mathfrak{S}}$-equivalences, $u$ is a termwise $\mathcal{T}$-equivalence (resp. a $\mathcal{T}^{\mathfrak{S}}$-equivalence) if and only if $v$ has the same property. But as $M_{*}$ and $L_{*}$ are

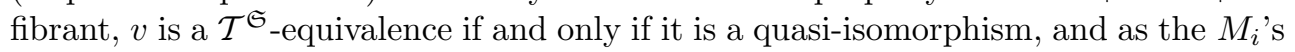
and the $N_{i}$ 's are fibrant, $v$ is a termwise $\mathcal{T}$-equivalence if and only if its is a termwise quasi-isomorphism. But we know that the evaluation functors are exact and form a conservative family of functors, so we see that $v$ is a termwise quasi-isomorphism if and only if it is a quasi-isomorphism. Hence we deduce that $u$ is a $\mathcal{T}^{\mathfrak{S}}$-equivalence if and only if it is a termwise $\mathcal{T}$-equivalence. 
7.6.

We can now consider a $\mathcal{G}$-cofibrant complex $S$ of $\mathcal{A}$. We have a commutative monoid $\operatorname{Sym}(S)$ in the category of symmetric sequences of $\operatorname{Comp}(\mathcal{A})$ (see 7.4). We will write $\operatorname{Sp}_{S}^{\mathfrak{S}}(\mathcal{A})$ for the category of symmetric $S$-spectra (i.e. of $\operatorname{Sym}(S)$-modules) in $\operatorname{Comp}(\mathcal{A})^{\mathfrak{S}}$; see 7.4 again. The category of symmetric $S$-spectra is a closed symmetric monoidal category (as any category of modules over a commutative monoid in a symmetric monoidal category; see e.g. [16]). We will write $\otimes_{\text {Sym }(S)}$ or simply $\otimes$ for the tensor product in $\operatorname{Sp}_{S}^{\mathfrak{S}}(\mathcal{A})$, and $\mathbf{1}_{S}$ for the unit (i.e. $\mathbf{1}_{S}=\operatorname{Sym}(S)$ as a symmetric sequence). We have a symmetric monoidal functor

$$
\operatorname{Comp}(\mathcal{A})^{\mathfrak{S}} \longrightarrow \operatorname{Sp}_{S}^{\mathfrak{S}}(\mathcal{A}), \quad A_{*} \longmapsto \operatorname{Sym}(S) \otimes^{\mathfrak{S}} A_{*},
$$

which has a right adjoint that consists to forget the action of $\operatorname{Sym}(S)$

$$
U: \operatorname{Sp}_{S}^{\mathfrak{S}}(\mathcal{A}) \longrightarrow \operatorname{Comp}(\mathcal{A})^{\mathfrak{S}}, \quad E \longmapsto E_{*} .
$$

If we think of symmetric $S$-spectra as collections $E=\left(E_{n}, \sigma_{n}\right)_{n \geqslant 0}$, then $U(E)$ is the symmetric sequence $E_{*}=\left(E_{n}\right)_{n \geqslant 0}$.

A morphism of symmetric $S$-spectra will be said to be a $\mathcal{T}$-equivalence (resp. a $\mathcal{T}$-fibration) if its image by $U$ is a termwise $\mathcal{T}$-equivalence (resp. a termwise fibration with respect to the $\mathcal{G}$-local model structure associated to $\mathcal{T}$ on $\left.\operatorname{Comp}(\mathcal{A})^{8}\right)$.

Proposition 7.9. The category $\operatorname{Sp}_{S}^{\mathfrak{S}}(\mathcal{A})$ is a proper cellular symmetric monoidal model category with the $\mathcal{T}$-equivalences (resp. the $\mathcal{T}$-fibrations) as weak equivalences (resp. as fibrations). Furthermore, if $\mathcal{G}$ is flat, then the monoid axiom holds in $\operatorname{Sp}_{S}^{\mathfrak{S}}(\mathcal{A})$.

Proof. The first assertion is proved by Hovey [14, Theorems 8.2 and 8.3]. The right properness follows from the fact that the $\mathcal{G}^{\mathfrak{S}}$-model structure associated to $\mathcal{T}^{\mathfrak{S}}$ on $\operatorname{Comp}(\mathcal{A})^{\mathfrak{S}}$ is proper and the fact that the functor $U$ preserves and detect weak equivalences, fibrations and pullbacks. We get the left properness in a similar way: this follows from Proposition 4.5 applied to $\mathcal{A}^{\mathfrak{S}}$ and from the facts that the cofibrations of $\operatorname{Sp}_{S}^{\mathfrak{S}}(\mathcal{A})$ are monomorphisms and that the functor $U$ preserves and detects weak equivalences, monomorphisms and pushouts. The last assertion is an application of $[20$, Theorem 4.1].

\section{7.}

We denote by $D_{\mathcal{T}}(\mathcal{A}, S)$ the localization of the category of symmetric $S$-spectra by the termwise $\mathcal{T}$-equivalences.

Proposition 7.10. The category $\operatorname{Sp}_{S}^{\mathfrak{S}}(\mathcal{A})$ is a proper cellular model category with the $\mathcal{T}$-equivalences (resp. the monomorphisms) as weak equivalences (resp. as cofibrations).

Proof. It follows from Propositions 4.5 and 4.6 that the class of monomorphisms which are $\mathcal{T}$-equivalences is closed under pushouts, transfinite compositions and

\footnotetext{
${ }^{8}$ The notion of $\mathcal{T}$-fibration depends on $\mathcal{T}$ and $\mathcal{G}$, but we will neglect this as we will always work
} with a fixed set $\mathcal{G}$ of generators. 
retracts. As the class of $\mathcal{T}$-equivalences is the class of weak equivalences of a cofibrantly generated model category, it is accessible. We thus obtain the expected model category structure by a straight application of Jeff Smith's theorem; see [3]. The left properness comes from the fact that any object is cofibrant for this model structure. The right properness comes from the fact that any fibration for this model structure is a $\mathcal{T}$-fibration and from the right properness of the model structure of Proposition 7.9 .

Proposition 7.11. If $\mathcal{G}$ is flat, then the functor $A_{*} \longmapsto \operatorname{Sym}(S) \otimes \mathfrak{S} A_{*}$ preserves termwise $\mathcal{T}$-equivalences.

Proof. As $S$ is $\mathcal{G}$-cofibrant, for any integer $n \geqslant 0, S^{\otimes n}$ is $\mathcal{G}$-cofibrant as well. This implies that the functor $A \longmapsto S^{\otimes n} \otimes A$ preserves $\mathcal{T}$-equivalences in $\operatorname{Comp}(\mathcal{A})$ : to see this, just remember that the canonical map $S^{\otimes n} \otimes^{\mathbf{L}} A \longrightarrow S^{\otimes n} \otimes A$ is an isomorphism in $D_{\mathcal{T}}(\mathcal{A})$ (see 3.8 and the last assertion of 4.11). This proposition thus follows from the construction of $\operatorname{Sym}(S) \otimes{ }^{\mathfrak{S}} A_{*}$ (see 7.2) and from the fact that $\mathcal{T}$ equivalences are stable by finite direct sums.

Remark 7.12. The model categories of Propositions 7.9 and 7.10 are stable. This means that a commutative square in the category of symmetric $S$-spectra is a homotopy pushout square if and only if it is a homotopy pullback square. In particular, the homotopy category $D_{\mathcal{T}}(\mathcal{A}, S)$ (localization of $\operatorname{Sp}_{S}^{\mathfrak{S}}(\mathcal{A})$ by the termwise $\mathcal{T}$ equivalences) is canonically endowed with a symmetric monoidal triangulated category structure, and the functor

$$
D_{\mathcal{T} \mathfrak{S}}\left(\mathcal{A}^{\mathfrak{S}}\right) \longrightarrow D_{\mathcal{T}}(\mathcal{A}, S), \quad A_{*} \longmapsto \operatorname{Sym}(S) \otimes \otimes^{\mathfrak{S}} A_{*}
$$

is a symmetric monoidal functor.

\section{8 .}

We have a symmetric monoidal functor

$$
\Sigma^{\infty}: \operatorname{Comp}(\mathcal{A}) \longrightarrow \operatorname{Sp}_{S}^{\mathfrak{S}}(\mathcal{A}), \quad A \longmapsto \operatorname{Sym}(S) \otimes^{\mathfrak{S}} A\{0\} .
$$

In particular, we have the formula

$$
\Sigma^{\infty}(A \otimes B) \simeq \Sigma^{\infty}(A) \otimes \Sigma^{\infty}(B) .
$$

The functor $\Sigma^{\infty}$ is a left adjoint of the functor

$$
\Omega^{\infty}: \operatorname{Sp}_{S}^{\mathfrak{S}}(\mathcal{A}) \longrightarrow \operatorname{Comp}(\mathcal{A}), \quad\left(E_{n}, \sigma_{n}\right)_{n \geqslant 0} \longmapsto E_{0} .
$$

These two functors form a Quillen adjunction if we consider the $\mathcal{G}$-model structure associated to $\mathcal{T}$ on $\operatorname{Comp}(\mathcal{A})$ and the model structure of Proposition 7.9 on $\operatorname{Sp}_{S}^{\mathfrak{S}}(\mathcal{A})$. We now introduce a very ugly abuse of notation. For an object $A$ of $\operatorname{Comp}(\mathcal{A})$ and a symmetric $S$-spectrum $E$, we define $E \otimes A$ to be $E \otimes \Sigma^{\infty}(A)$. This can be rewritten as

$$
E \otimes A=E \otimes_{\operatorname{Sym}(S)}\left(\operatorname{Sym}(S) \otimes^{\mathfrak{S}} A\{0\}\right) \simeq E \otimes^{\mathfrak{S}} A\{0\} .
$$

In terms of collections $E=\left(E_{n}, \sigma_{n}\right)_{n \geqslant 0}$, we have $E \otimes A=\left(E_{n} \otimes A, \sigma_{n} \otimes 1_{A}\right)_{n \geqslant 0}$. This makes our purpose more precise: we would like the functor $E \longmapsto E \otimes S$ to 
be a left Quillen equivalence. This is not the case in general, so that we have to localize a little further.

We will say that a symmetric $S$-spectrum $E=\left(E_{n}, \sigma_{n}\right)_{n \geqslant 0}$ is an $\Omega^{\infty}$-spectrum if for any integer $n \geqslant 0, E_{n}$ is a $\mathcal{G}$-local and $\mathcal{T}$-local complex of $\mathcal{A}$ and the map induced by adjunction from $\sigma_{n}$

$$
\tilde{\sigma}_{n}: E_{n} \longrightarrow \operatorname{Hom}\left(S, E_{n+1}\right)
$$

is a quasi-isomorphism (here Hom is the internal Hom of the category of complexes of $\mathcal{A}$ ). We remark that as $S$ is $\mathcal{G}$-cofibrant and as $E_{n+1}$ is a fibrant object of the $\mathcal{G}$-model structure associated to $\mathcal{T}, \operatorname{Hom}\left(S, E_{n+1}\right)$ is also fibrant. This implies that the map 7.5 is a quasi-isomorphism if and only if it is a $\mathcal{T}$-equivalence.

A morphism of symmetric $S$-spectra $u: A \longrightarrow B$ is a stable $\mathcal{T}$-equivalence if for any $\Omega^{\infty}$-spectrum $E$, the induced map

$$
u^{*}: \operatorname{Hom}_{D_{\mathcal{T}}(\mathcal{A}, S)}(B, E) \longrightarrow \operatorname{Hom}_{D_{\mathcal{T}}(\mathcal{A}, S)}(A, E)
$$

is an isomorphism of abelian groups. For example, any termwise $\mathcal{T}$-equivalence is a stable $\mathcal{T}$-equivalence.

A morphism of symmetric $S$-spectra is a stable $\mathcal{G}$-cofibration is it is a cofibration of the model structure of Proposition 7.9.

A morphism of symmetric $S$-spectra is a stable $\mathcal{T}$-fibration if it has the right lifting property with respect to the stable $\mathcal{G}$-cofibrations which are also stable $\mathcal{T}$ equivalences.

Proposition 7.13. The category of symmetric $S$-spectra is a stable proper cellular symmetric monoidal model category with the stable $\mathcal{T}$-equivalences as weak equivalences, the stable $\mathcal{G}$-cofibrations as cofibrations, and the stable $\mathcal{T}$-fibrations as fibrations. Moreover, the stable $\mathcal{T}$-fibrations are the termwise $\mathcal{G}$-surjective morphisms $p: E \longrightarrow F$ such that $\operatorname{ker} p$ is an $\Omega^{\infty}$-spectrum. In particular, the fibrant objects of this model structure are the $\Omega^{\infty}$-spectra.

Proof. It follows from [14, Theorem 8.11] that we have defined a model category structure. As this model structure is by definition a left Bousfield localization of a left proper cellular model category, it is left proper. This model structure is stable as the stable $\mathcal{T}$-equivalences are the maps inducing isomorphisms on the cohomological functors $\operatorname{Hom}_{D_{\mathcal{T}}(\mathcal{A}, S)}(?, E)$ with $E$ any $\Omega^{\infty}$-spectrum. The right properness follows then from the right properness of the model category of Proposition 7.9 as follows: Consider a pullback square of symmetric spectra

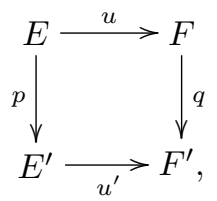

where $p$ is a stable $\mathcal{T}$-fibration. As $p$ is also a fibration for the model structure of Proposition 7.9, this square is a homotopy pullback square. But as this latter model structure is stable, this square is also a homotopy pushout square. This implies that this square is a homotopy pushout square in the model category defined by the stable $\mathcal{T}$-equivalences. Again, as this latter model structure is stable, we see that 
this commutative square has to be a homotopy pullback square. Hence, if moreover $u^{\prime}$ is a stable $\mathcal{T}$-equivalence, then $u$ has to be a stable $\mathcal{T}$-equivalence as well, and we have proved the right properness. The characterization of the stable $\mathcal{T}$-fibrations starts with the description of the fibrant objects as the $\Omega^{\infty}$-spectra which comes from [14, Theorem 8.8]. We can then finish the proof using the fact that the fibrations of the model structure of Proposition 7.9 are exactly the termwise $\mathcal{G}$-surjective maps with termwise $\mathcal{G}$-local and $\mathcal{T}$-local kernels (see 5.5) and reproducing the proof of Proposition 4.7 .

\section{9 .}

The model structure of Proposition 7.13 will be called the $S$-stable model structure associated to $\mathcal{T}$. We denote by $D_{\mathcal{T}}\left(\mathcal{A}, S^{-1}\right)$ the localization of the category $\operatorname{Sp}_{S}^{\mathfrak{S}}(\mathcal{A})$ by the stable $\mathcal{T}$-equivalences.

7.10.

It remains to show that the functor $E \longmapsto E \otimes S=E \otimes \Sigma^{\infty}(S)$ is a left Quillen equivalence. We already know that it is a left Quillen functor with respect to the $S$-stable model category structure associated to $\mathcal{T}$. We write

$$
D_{\mathcal{T}}\left(\mathcal{A}, S^{-1}\right) \longrightarrow D_{\mathcal{T}}\left(\mathcal{A}, S^{-1}\right), \quad E \longmapsto E \otimes^{\mathbf{L}} S
$$

for the corresponding total left derived functor. We define the symmetric $S$-spectra $S^{-1}=\operatorname{Sym}(S) \otimes^{\mathfrak{S}} S\{-1\}$. The tensor product by $S^{-1}$,

$$
\operatorname{Sp}_{S}^{\mathfrak{S}}(\mathcal{A}) \longrightarrow \operatorname{Sp}_{S}^{\mathfrak{S}}(\mathcal{A}), \quad E \longmapsto E \otimes S^{-1} \simeq E \otimes^{\mathfrak{S}} S\{-1\},
$$

is also a left Quillen functor and has a total left derived functor

$$
D_{\mathcal{T}}\left(\mathcal{A}, S^{-1}\right) \longrightarrow D_{\mathcal{T}}\left(\mathcal{A}, S^{-1}\right), \quad E \longmapsto E \otimes^{\mathbf{L}} S^{-1}
$$

Note that, if moreover $\mathcal{G}$ is flat, as both $\Sigma^{\infty}(S)$ and $S^{-1}$ are stably $\mathcal{G}$-cofibrant, for any symmetric $S$-spectrum $E$, we have canonical isomorphisms in $D_{\mathcal{T}}\left(\mathcal{A}, S^{-1}\right)(7.22)$

$$
E \otimes^{\mathbf{L}} S \simeq E \otimes S \text { and } E \otimes^{\mathbf{L}} S^{-1} \simeq E \otimes S^{-1}
$$

(but this property will not be needed).

Proposition 7.14. The functors $E \longmapsto E \otimes^{\mathbf{L}} S$ and $E \longmapsto E \otimes^{\mathbf{L}} S^{-1}$ are equivalences of categories and are quasi-inverse to each other. In particular, the functor $E \longmapsto E \otimes S=E \otimes \Sigma^{\infty}(S)$ is a left Quillen equivalence.

Proof. See [14, Theorem 8.10].

Example 7.15. Consider the notation and conventions of 4.12.

Let $\mathbb{G}_{m}$ be the multiplicative group scheme over $S$. It is a pointed scheme with $S$-point the unit section $s$. We associate to this pointed scheme a sheaf with transfers $\tilde{L}_{S}\left(\mathbb{G}_{m}\right)$, defined as the cokernel of the split monomorphism $L_{S}(S) \stackrel{s_{*}}{\longrightarrow} L_{S}\left(\mathbb{G}_{m}\right)$.

A symmetric $\tilde{L}_{S}\left(\mathbb{G}_{m}\right)$-spectrum in the category $\operatorname{Comp}(\mathcal{A})^{\mathfrak{S}}$ (cf. 7.6 ) will be called a motivic spectrum over $S$.

According to Proposition 7.13, the category of symmetric Tate spectra $S p_{\text {Tate }}(S)$ over $S$ is a stable symmetric monoidal category. We denote its homotopy category by $D M(S)$. 
By construction, we obtain a pair of adjoint functors

$$
\mathbf{L} \Sigma^{\infty}: D M^{e f f}(S) \rightleftarrows D M(S): \mathbf{R} \Omega^{\infty}
$$

such that $\mathbf{L} \Sigma^{\infty}$ is symmetric monoidal, and $\mathbf{L} \Sigma^{\infty} \tilde{M}_{S}\left(\mathbb{G}_{m}\right)$ (usually denoted by $\left.\mathbb{Z}_{S}(1)[1]\right)$ is invertible (cf. 7.14$)^{9}$.

Using an obvious extension of the base change (resp. forget the base) functor from complexes to spectra, and applying the obvious analog of Proposition 4.9, we can construct for any morphism of regular schemes $f: T \longrightarrow S$ a pair of adjoint functors

$$
\mathbf{L} f^{*}: D M(S) \rightleftarrows D M(T): \mathbf{R} f_{*}
$$

and in the case where $f$ is smooth another pair

$$
\mathbf{L} f_{\sharp}: D M(S) \rightleftarrows D M(T): \mathbf{R} f^{*}=f^{*} .
$$

This construction will be generalized and studied more closely in the forthcoming paper [6].

\subsection{1 .}

We will need a few more details about the relationship between the tensor product by $S$ and $S^{-1}$. We will write $\mathbf{H o m}_{\mathfrak{S}}$ and $\mathbf{H o m}_{S}$ for the internal Hom's in the categories of symmetric sequences and of symmetric $S$-spectra respectively.

Let $E=\left(E_{n}, \sigma_{n}\right)_{n \geqslant 0}$ be a symmetric $S$-spectrum. One can define the symmetric $S$-spectrum $E\{1\}$ by

$$
E\{1\}=\operatorname{Hom}_{\mathfrak{S}}(\mathbf{1}\{-1\}, E) \simeq \operatorname{Hom}_{S}(\operatorname{Sym}(S)\{-1\}, E) .
$$

In other words, $E\{1\}=\left(E\{1\}_{n}, \sigma\{1\}_{n}\right)_{n \geqslant 0}$, where for $n \geqslant 0$, we have $E\{1\}_{n}=E_{n+1}$ and $\sigma\{1\}_{n}=\sigma_{n+1}$, and the action of $\mathfrak{S}_{n}$ on $E_{n+1}$ is induced by the obvious inclusion $\mathfrak{S}_{n} \subset \mathfrak{S}_{n+1}$. The maps $\sigma_{n}$ define a morphism of symmetric $S$-spectra called the suspension map

$$
\sigma: E \otimes S \longrightarrow E\{1\}
$$

By adjunction, this defines a map

$$
\tilde{\sigma}: E \longrightarrow \operatorname{Hom}_{S}\left(\Sigma^{\infty}(S), E\{1\}\right) .
$$

But $\operatorname{Hom}_{S}\left(\Sigma^{\infty}(S), E\{1\}\right) \simeq \operatorname{Hom}_{S}\left(\operatorname{Sym}(S) \otimes^{\mathfrak{S}} S\{-1\}, E\right)$, so that we have a map

$$
\tilde{\sigma}: E \longrightarrow \operatorname{Hom}_{S}\left(\operatorname{Sym}(S) \otimes^{\mathfrak{S}} S\{-1\}, E\right) .
$$

Then $E$ is an $\Omega^{\infty}$-spectrum if and only if it is termwise $\mathcal{G}$-local and $\mathcal{T}$-local and if the map (7.8) is a termwise $\mathcal{T}$-equivalence (this is an easy translation of the definition). We deduce from this and from the fact that the functor ? $\otimes S$ preserves stable $\mathcal{T}$ equivalences (see 7.22) that for any $\Omega^{\infty}$-spectrum $E$, the map (7.6) is a stable $\mathcal{T}$ equivalence $^{10}$. As $E\{1\}$ is then still an $\Omega^{\infty}$-spectrum, we can iterate this process and obtain for any $\Omega^{\infty}$-spectrum $E$ a canonical stable $\mathcal{T}$-equivalence

$$
\sigma^{n}: E \otimes S^{\otimes n}=E \otimes \Sigma^{\infty}(S)^{\otimes n} \longrightarrow E\{n\} .
$$

\footnotetext{
${ }^{9}$ In fact, we can state a universal property satisfied by $D M(S)$ from these two properties.

${ }^{10}$ The proof of Proposition 7.14 follows from this fact once we have noticed that $\operatorname{Hom}_{S}(\operatorname{Sym}(S) \otimes \mathfrak{S}$ $S\{-1\}, ?)$ is a right adjoint to the functor ? $\otimes S^{-1}$.
} 


\subsection{2 .}

The functor $\Sigma^{\infty}: \operatorname{Comp}(\mathcal{A}) \longrightarrow \operatorname{Sp}_{S}^{\mathfrak{S}}(\mathcal{A})$ is a left Quillen functor from the $\mathcal{G}$-model structure associated to $\mathcal{T}$ to the $S$-stable model structure associated to $\mathcal{T}$ (this is immediate). If moreover $\mathcal{G}$ is flat, then it easily follows from Proposition 7.11 that it sends $\mathcal{T}$-equivalences to stable $\mathcal{T}$-equivalences.

The fact $\Sigma^{\infty}$ is a left Quillen functor implies that its right adjoint $\Omega^{\infty}$ is a right Quillen functor and that the total left derived functor

$$
\mathbf{L} \Sigma^{\infty}: D_{\mathcal{T}}(\mathcal{A}) \longrightarrow D_{\mathcal{T}}\left(\mathcal{A}, S^{-1}\right)
$$

is a left adjoint of the total right derived functor of $\Omega^{\infty}$

$$
\mathbf{R} \Omega^{\infty}: D_{\mathcal{T}}\left(\mathcal{A}, S^{-1}\right) \longrightarrow D_{\mathcal{T}}(\mathcal{A}) .
$$

In particular, for any complex $X$ of $\mathcal{A}$ and any symmetric $S$-spectrum $E$, we have canonical isomorphisms of abelian groups

$$
\operatorname{Hom}_{D_{\mathcal{T}}\left(\mathcal{A}, S^{-1}\right)}\left(\mathbf{L} \Sigma^{\infty}(X), E\right) \simeq \operatorname{Hom}_{D_{\mathcal{T}}(\mathcal{A})}\left(X, \mathbf{R} \Omega^{\infty}(E)\right) .
$$

Moreover, for any $\Omega^{\infty}$-spectrum $E$, we have $\mathbf{R} \Omega^{\infty}(E)=E_{0}$.

A weak $\Omega^{\infty}$-spectrum is a symmetric $S$-spectrum $E=\left(E_{n}, \sigma_{n}\right)_{n \geqslant 0}$ such that for any $n \geqslant 0$, the map

$$
E_{n} \longrightarrow \mathbf{R H o m}\left(S, E_{n+1}\right)
$$

obtained by adjunction in $D_{\mathcal{T}}(\mathcal{A}, S)$ from the map $\sigma_{n}: E_{n} \otimes S \longrightarrow E_{n+1}$ is an isomorphism. It is obvious that any $\Omega^{\infty}$-spectrum is a weak $\Omega^{\infty}$-spectrum.

Proposition 7.16. Let $E$ be a weak $\Omega^{\infty}$-spectrum and $n \geqslant 0$ an integer. Then we have canonical isomorphisms $\mathbf{R} \Omega^{\infty}\left(E \otimes S^{\otimes n}\right) \simeq E_{n}$ in $D_{\mathcal{T}}(\mathcal{A})$. In other words, for any object $X$ of $D_{\mathcal{T}}(\mathcal{A})$, we have canonical isomorphisms

$$
\begin{aligned}
\operatorname{Hom}_{D_{\mathcal{T}}(\mathcal{A})}\left(X, E_{n}\right) & \simeq \operatorname{Hom}_{D_{\mathcal{T}}(\mathcal{A})}\left(X, \mathbf{R} \Omega^{\infty}\left(E \otimes S^{\otimes n}\right)\right) \\
& \simeq \operatorname{Hom}_{D_{\mathcal{T}}\left(\mathcal{A}, S^{-1}\right)}\left(\mathbf{L} \Sigma^{\infty}(X), E \otimes S^{\otimes n}\right) .
\end{aligned}
$$

Moreover, the iterated suspension map $\sigma^{n}: E \otimes S^{\otimes n} \longrightarrow E\{n\}$ is an isomorphism in $D_{\mathcal{T}}\left(\mathcal{A}, S^{-1}\right)$.

Proof. We know the assertion is true when $E$ is actually an $\Omega^{\infty}$-spectrum (see 7.11). In general, thanks to the existence of the model structure of Proposition 7.9, one can choose a termwise $\mathcal{T}$-equivalence $E \longrightarrow E^{\prime}$ such that $E^{\prime}$ is termwise $\mathcal{G}$-local and $\mathcal{T}$ local. But $E^{\prime}$ is also a weak $\Omega^{\infty}$-spectrum, and this implies immediately that $E^{\prime}$ is an $\Omega^{\infty}$-spectrum. As the maps $E_{i} \longrightarrow E_{i}^{\prime}$ are $\mathcal{T}$-equivalences for any $i \geqslant 0$, this implies that $E\{n\} \longrightarrow E^{\prime}\{n\}$ is also a termwise $\mathcal{T}$-equivalence for any integer $n$. Hence we are reduced to the $\Omega^{\infty}$-spectrum case.

Proposition 7.17. A pushout square of symmetric S-spectra,

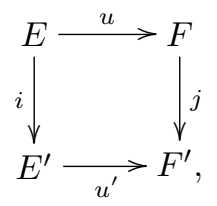

in which $i$ is a monomorphism, is a homotopy pushout square with respect to the 
$S$-stable model structure associated to $\mathcal{T}$. In particular, if $i$ (resp. $u$ ) is a stable $\mathcal{T}$ equivalence, then $j$ (resp. $\left.u^{\prime}\right)$ is a stable $\mathcal{T}$-equivalence.

Proof. It is sufficient to prove that this commutative square is a homotopy pushout square for the model structure of Proposition 7.9. This square is obviously a homotopy pushout square for the model structure of Proposition 7.10, which implies that it has to be a homotopy pushout square for the model structure of Proposition 7.9 as well.

Proposition 7.18. The stable $\mathcal{T}$-equivalences are stable by filtering colimits in $\operatorname{Sp}_{S}^{\mathfrak{S}}(\mathcal{A})$.

Proof. We know that the termwise $\mathcal{T}$-equivalences are stable by filtering colimits (this follows immediately from 4.6). This is equivalent to say that for any filtering category $I$ and any functor $F$ from $I$ to $\operatorname{Sp}_{S}^{\mathfrak{S}}(\mathcal{A})$, the canonical map

$$
\underset{i}{\operatorname{holim}} F_{i} \longrightarrow \underset{i}{\lim } F_{i}
$$

is a termwise $\mathcal{T}$-equivalence, hence a stable $\mathcal{T}$-equivalence. But as the functor holim sends stable $\mathcal{T}$-equivalences to stable $\mathcal{T}$-equivalences, this achieves the proof.

Proposition 7.19. The category of symmetric S-spectra is endowed with a cellular proper model category structure with the stable $\mathcal{T}$-equivalences as weak equivalences and the monomorphisms as cofibrations.

Proof. It follows from Propositions 7.17 and 7.18 that the class of monomorphisms which are stable $\mathcal{T}$-equivalences is stable by pushout, transfinite composition and retract. We thus obtain the expected model structure as a left Bousfield localization of the model structure of Proposition 7.10. The left properness is obvious (any object is cofibrant) and the right properness comes from the right properness of the $S$-stable model structure associated to $\mathcal{T}$ and from the fact that any fibration is, in particular, a fibration for the $S$-stable model structure.

\subsection{3 .}

The model structure of Proposition 7.19 will be called the injective $S$-stable model structure associated to $\mathcal{T}$.

From now on, we suppose that $\mathcal{G}$ is flat.

Lemma 7.20. Let $A_{*}$ be a $\mathcal{G}^{\mathfrak{S}}$-cofibrant symmetric sequence, and $E$ be a symmetric S-spectrum. Then the canonical map

$$
E \otimes^{\mathbf{L}}\left(\operatorname{Sym}(S) \otimes^{\mathfrak{S}} A_{*}\right) \longrightarrow E \otimes\left(\operatorname{Sym}(S) \otimes^{\mathfrak{S}} A_{*}\right)
$$

is an isomorphism in $D_{\mathcal{T}}(\mathcal{A}, S)$ (hence in $D_{\mathcal{T}}\left(\mathcal{A}, S^{-1}\right)$ as well).

Proof. We begin with the following observation: the functor

$$
\operatorname{Comp}(\mathcal{A})^{\mathfrak{S}} \longrightarrow \operatorname{Comp}(\mathcal{A})^{\mathfrak{S}}, \quad E \longmapsto E \otimes^{\mathfrak{S}} A_{*}
$$

preserves termwise $\mathcal{T}$-equivalences. This is because by virtue of Lemma $7.3, \mathcal{G}^{\mathfrak{S}}$ is a flat generating family of $\mathcal{A}^{\mathfrak{S}}$; see Remark 3.8 . 
Let now $E$ be a symmetric $S$-spectrum. We can choose a termwise $\mathcal{T}$-equivalence $E^{\prime} \longrightarrow E$ in $\operatorname{Sp}_{S}^{\mathfrak{S}}(\mathcal{A})$ with $E^{\prime}$ cofibrant for the model structure of Proposition 7.9. But then we have a termwise $\mathcal{T}$-equivalence (hence a stable $\mathcal{T}$-equivalence)

$$
E^{\prime} \otimes\left(\operatorname{Sym}(S) \otimes^{\mathfrak{S}} A_{*}\right) \simeq E^{\prime} \otimes^{\mathfrak{S}} A_{*} \longrightarrow E \otimes^{\mathfrak{S}} A_{*} \simeq E \otimes\left(\operatorname{Sym}(S) \otimes^{\mathfrak{S}} A_{*}\right) .
$$

This implies our assertion as both $E^{\prime}$ and $\operatorname{Sym}(S) \otimes{ }^{\mathfrak{G}} A_{*}$ are cofibrant symmetric $S$-spectra.

Lemma 7.21. Let $E$ be a symmetric $S$-spectrum. Then the functor

$$
\operatorname{Sp}_{S}^{\mathfrak{S}}(\mathcal{A}) \longrightarrow \operatorname{Sp}_{S}^{\mathfrak{S}}(\mathcal{A}), \quad F \longmapsto E \otimes F
$$

is a left Quillen functor from the model structure of Proposition 7.9 to the model structure of Proposition 7.10.

Proof. Proposition 3.4 applied to $\mathcal{A}^{\mathfrak{S}}$ gives the following: If $u: A_{*} \longrightarrow B_{*}$ is a $\mathcal{G}^{\mathfrak{S}}$ cofibration of symmetric sequences, then the induced map

$$
E \otimes\left(\operatorname{Sym}(S) \otimes^{\mathfrak{S}} A_{*}\right) \simeq E^{\prime} \otimes^{\mathfrak{S}} A_{*} \longrightarrow E \otimes^{\mathfrak{S}} B_{*} \simeq E \otimes\left(\operatorname{Sym}(S) \otimes^{\mathfrak{S}} B_{*}\right)
$$

is a monomorphism. If moreover $u$ is a quasi-isomorphism, then so is the induced map above. Hence the lemma is proved for $\mathcal{T}=\varnothing$. To prove the general case, it is sufficient to prove that the tensor product by $E$ sends the generating trivial cofibrations of the model category of Proposition 7.9 to termwise $\mathcal{T}$-equivalences. But the generating trivial cofibrations are all of shape

$$
\operatorname{Sym}(S) \otimes{ }^{\mathfrak{S}} A\{-i\} \longrightarrow \operatorname{Sym}(S) \otimes^{\mathfrak{S}} B\{-i\},
$$

where $i \geqslant 0$ is an integer, and $A \longrightarrow B$ is a $\mathcal{G}$-cofibration and a $\mathcal{T}$-equivalence in $\operatorname{Comp}(\mathcal{A})$. We conclude easily using Lemma 7.20.

Proposition 7.22. Let $E$ and $F$ be symmetric $S$-spectra. If $E$ or $F$ is stably $\mathcal{G}$ cofibrant (i.e. a cofibrant object of the model category of Proposition 7.9), then the canonical map

$$
E \otimes \mathbf{L} F \longrightarrow E \otimes F
$$

is an isomorphism in $D_{\mathcal{T}}(\mathcal{A}, S)$ (hence in $D_{\mathcal{T}}\left(\mathcal{A}, S^{-1}\right)$ as well).

Proof. Let $E$ be a fixed symmetric $S$-spectrum. This object gives rise to two triangulated endofunctors of the category $D_{\mathcal{T}}(\mathcal{A}, S)$. We denote the first one by $\Phi$. It is defined by $\Phi(F)=E \otimes^{\mathbf{L}} F$ (where we insist that $\otimes^{\mathbf{L}}$ is the total left derived functor of the left Quillen bifunctor $\otimes$ on $\operatorname{Sp}_{S}^{\mathfrak{S}}(\mathcal{A})$ ). The second one, denoted by $\Psi$ is the total left derived functor of the functor $F \longmapsto E \otimes F$ (which is well defined by virtue of Lemma 7.21). We have a canonical map of triangulated functors $\Phi \longrightarrow \Psi$, and we would like it to be an isomorphism. By Lemma 7.20, we already know that it induces an isomorphism on objects of type $\operatorname{Sym}(S) \otimes{ }^{\mathfrak{S}} A_{*}$ for any $\mathcal{G}^{\mathfrak{S}}$-cofibrant symmetric sequence $A_{*}$. But as the cokernels of the generating cofibrations of the model structure of Proposition 7.9 are all of this type, it follows from [12, Theorem 7.3.1] that the objects of shape $\operatorname{Sym}(S) \otimes{ }^{\mathfrak{S}} A_{*}$ form a generating family of the triangulated category $D_{\mathcal{T}}(\mathcal{A}, S)$. This means that the smallest localizing subcategory of $D_{\mathcal{T}}(\mathcal{A}, S)$ that contains the $\operatorname{Sym}(S) \otimes{ }^{\mathfrak{S}} A_{*}$ 's is $D_{\mathcal{T}}(\mathcal{A}, S)$ itself. This implies that $\Phi \longrightarrow \Psi$ is an 
isomorphism: it remains to prove that the full subcategory of $D_{\mathcal{T}}(\mathcal{A}, S)$ that consists of object $F$ such that $\Phi(F) \simeq \Psi(F)$ is a triangulated subcategory and is stable by direct sums; which is obvious. But if $F$ is stably $\mathcal{G}$-cofibrant, the map $\Phi(F) \longrightarrow \Psi(F)$ is just the map $E \otimes^{\mathbf{L}} F \longrightarrow E \otimes F$.

Proposition 7.23. For any symmetric $S$-spectrum $E$, the functor $A \longmapsto A \otimes E$ is a left Quillen functor from the $S$-stable model structure associated to $\mathcal{T}$ to the injective $S$-stable model structure associated to $\mathcal{T}$.

Proof. This follows immediately from Lemmata 4.8 and 7.21 and from Proposition 7.22 .

Corollary 7.24. If $\mathcal{G}$ is flat, then the $S$-stable model category structure associated to $\mathcal{T}$ satisfies the monoid axiom of [20].

Proof. This is an immediate consequence of Proposition 7.23.

\subsection{4 .}

We still assume that $\mathcal{G}$ is flat.

An $S$-ring spectrum, or simply a ring spectrum is a commutative monoid in the category of symmetric $S$-spectra. Such a ring spectrum is commutative if it is commutative as a monoid.

If $\mathcal{E}$ is a ring spectrum, we write $\operatorname{Sp}_{S}^{\mathfrak{S}}(\mathcal{A}, \mathcal{E})$ for the category of left $\mathcal{E}$-modules. If moreover $\mathcal{E}$ is commutative, the category $\operatorname{Sp}_{S}^{\mathfrak{S}}(\mathcal{A}, \mathcal{E})$ is a closed symmetric monoidal category. We have a functor

$$
\operatorname{Sp}_{S}^{\mathfrak{S}}(\mathcal{A}) \longrightarrow \operatorname{Sp}_{S}^{\mathfrak{S}}(\mathcal{A}, \mathcal{E}), \quad F \longmapsto \mathcal{E} \otimes F
$$

which is a left adjoint to the forgetful functor

$$
\operatorname{Sp}_{S}^{\mathfrak{S}}(\mathcal{A}, \mathcal{E}) \longrightarrow \operatorname{Sp}_{S}^{\mathfrak{S}}(\mathcal{A}), \quad M \longmapsto M
$$

If $\mathcal{E}$ is commutative, the functor $F \longmapsto \mathcal{E} \otimes F$ is of course symmetric monoidal. A morphism of $\mathcal{E}$-modules is a stable $\mathcal{T}$-equivalence (resp. a stable $\mathcal{T}$-fibration) if it is so as a morphism of symmetric $S$-spectra.

We will write $D_{\mathcal{T}}(\mathcal{A}, \mathcal{E})$ for the localization of the category of $\mathcal{E}$-modules by the class of stable $\mathcal{T}$-equivalences.

Corollary 7.25. Let $\mathcal{E}$ be a ring spectrum. Then the category of left $\mathcal{E}$-modules in $\operatorname{Sp}_{S}^{\mathfrak{S}}(\mathcal{A})$ is a cellular proper stable model category with the stable $\mathcal{T}$-equivalences as weak equivalences and the stable $\mathcal{T}$-fibrations as fibrations. If moreover $\mathcal{E}$ is commutative, then this is a symmetric monoidal model category which satisfies the monoid axiom.

Proof. The fact that we get a cofibrantly generated model category (symmetric monoidal and satisfying the monoid axiom if $\mathcal{E}$ is commutative) comes from [20, Theorem 4.1]. The proof of properness and of stability is left to the reader (it is the same as in the proof of 7.9 ). 


\section{References}

[1] M. Artin, A. Grothendieck, and J.-L. Verdier, Théorie des topos et cohomologie étale des schémas, Lecture Notes in Math., vol. 269, 270, 305, Springer-Verlag, New York, 1972. Sém. de Géom. Alg. du Bois-Marie 1963-64 (SGA 4).

[2] P. Balmer and M. Schlichting, Idempotent completion of triangulated categories, J. Algebra 236 (2001), no. 2, 819-834.

[3] T. Beke, Sheafifiable homotopy model categories, Math. Proc. Cambridge Philos. Soc. 129 (2000), no. 3, 447-475.

[4] K.S. Brown, Abstract homotopy theory and generalized sheaf cohomology, Trans. Amer. Math. Soc. 186 (1974), 419-458.

[5] J.D. Christensen and M. Hovey, Quillen model structures for relative homological algebra, Math. Proc. Cambridge Philos. Soc. 133 (2002), no. 2, 261-293.

[6] D.-C. Cisinski and F. Déglise, Triangulated categories of motives. In preparation.

[7] D.-C. Cisinski and F. Déglise, Mixed Weil cohomologies, 2007. Preprint.

[8] F. Déglise, Finite correspondances and transfers over a regular base, London Mathematical Society Lecture Note Series, vol. 343, Cambridge University Press, Cambridge, Algebraic cycles and motives. Volume 1. Selected papers of the EAGER conference (Leiden, Netherlands, Aug. 30-Sept. 3, 2004) on the occasion of the 75th birthday of Professor J.P. Murre, 2007.

[9] E.M. Friedlander, A. Suslin, and V. Voevodsky, Cycles, transfers and motivic homology theories, Annals of Mathematics Studies, vol. 143, Princeton Univ. Press, Princeton, NJ, 2000.

[10] P. Gabriel and N. Popescu, Caractérisation des catégories abéliennes avec générateurs et limites inductives exactes, C. R. Acad. Sci. Paris 258 (1964), $4188-4190$.

[11] P.S. Hirschhorn, Model categories and their localizations, Math. Surveys and Monographs, vol. 99, Amer. Math. Soc., Providence, RI, 2003.

[12] M. Hovey, Model categories, Math. Surveys and Monographs, vol. 63, Amer. Math. Soc., Providence, RI, 1999.

[13] M. Hovey, Model category structures on chain complexes of sheaves, Trans. Amer. Math. Soc. 353 (2001), no. 6, 2441-2457.

[14] M. Hovey, Spectra and symmetric spectra in general model categories, J. Pure Appl. Alg. 165 (2001), no. 1, 63-127.

[15] W. Lowen, A generalization of the Gabriel-Popescu theorem, J. Pure Appl. Algebra 190 (2004), no. 1-3, 197-211.

[16] S. MacLane, Categories for the working mathematician, Second edition, Graduate Texts in Math., vol. 5, Springer-Verlag, New York, 1998.

[17] B. Mitchell, A quick proof of the Gabriel-Popescu theorem, J. Pure Appl. Algebra 20 (1981), no. 3, 313-315.

[18] F. Morel and V. Voevodsky, $\mathbb{A}^{1}$-homotopy theory of schemes, Publ. Math. IHES 90 (1999), 45-143. 
[19] A. Neeman, Triangulated categories, Annals of Math. Studies, vol. 148, Princeton Univ. Press, Princeton, NJ, 2001.

[20] S. Schwede and B. Shipley, Algebras and modules in monoidal model categories, Proc. London Math. Soc. 80 (2000), no. 2, 491-511.

[21] M. Spitzweck, Operads, algebras and modules in monoidal model categories and motives, 2001. Ph.D. thesis, Mathematisch Naturwissenschatlichen Fakultät, Rheinischen Friedrich Wilhelms Universität, Bonn.

Denis-Charles Cisinski cisinski@math.univ-paris13.fr

LAGA, CNRS (UMR 7539), Université Paris 13, Avenue Jean-Baptiste Clément, 93430 Villetaneuse, France

Frédéric Déglise deglise@math.univ-paris13.fr

LAGA, CNRS (UMR 7539), Université Paris 13, Avenue Jean-Baptiste Clément, 93430 Villetaneuse, France 